\title{
The Hunt for Red Quasars : Luminous Obscured Black Hole Growth Unveiled in the Stripe 82 X-Ray Survey
}

\section{LaMassa, Stephanie M.}

2017-10-01

LaMassa , S M , Glikman , E, Brusa, M , Rigby , J R , Ananna , T T , Stern , D , Lira , P , Urry , C M , Salvato, M , Alexandroff , R , Allevato , V , Cardamone , C , Civano , F , Coppi , P , Farrah , D , Komossa , S , Lanzuisi , G, Marchesi , S, Richards , G , Trakhtenbrot , B \& Treister , E 2017 , ' The Hunt for Red Quasars : Luminous Obscured Black Hole Growth Unveiled in the Stripe 82 X-Ray Survey ' , Astrophysical Journal , vol. 847 , no. 2 , 100 . https://doi.org/10.3847/1538

http://hdl.handle.net/10138/226026

https://doi.org/10.3847/1538-4357/aa87b5

unspecified

publishedVersion

Downloaded from Helda, University of Helsinki institutional repository.

This is an electronic reprint of the original article.

This reprint may differ from the original in pagination and typographic detail.

Please cite the original version. 


\title{
The Hunt for Red Quasars: Luminous Obscured Black Hole Growth Unveiled in the Stripe $82 \mathrm{X}$-Ray Survey
}

Stephanie M. LaMassa ${ }^{1}$ (D), Eilat Glikman ${ }^{2}$ (D), Marcella Brusa ${ }^{3,4}$ (D), Jane R. Rigby ${ }^{5}$ (D), Tonima Tasnim Ananna ${ }^{6,7}$, Daniel Stern ${ }^{8}$ (D), Paulina Lira ${ }^{9}$, C. Megan Urry ${ }^{6,7}$ (D) , Mara Salvato $^{10}$ (D), Rachael Alexandroff ${ }^{11}$ (D), Viola Allevato ${ }^{12,13}$ (D), Carolin Cardamone ${ }^{14}$ (D), Francesca Civano $^{15}$, Paolo Coppi ${ }^{6,7}$, Duncan Farrah ${ }^{16}$ (D) S. Komossa ${ }^{17}$ (D), Giorgio Lanzuisi ${ }^{3,4}$ (D), Stefano Marchesi ${ }^{18}$ (D),

Gordon Richards $^{19}$ (D), Benny Trakhtenbrot ${ }^{20}$ (D), and Ezequiel Treister ${ }^{21}$

${ }^{1}$ Space Telescope Science Institute, 3700 San Martin Drive, Baltimore MD, 21218, USA

${ }^{2}$ Department of Physics, Middlebury College, Middlebury, VT 05753, USA

${ }^{3}$ INAF-Osservatorio Astronomico di Bologna, via Ranzani 1, I-40127 Bologna, Italy

${ }^{4}$ Dipartimento di Fisica e Astronomia, Universita' di Bologna, viale Berti Pichat 6/2, I-40127 Bologna, Italy

${ }^{5}$ Observational Cosmology Lab, NASA Goddard Space Flight Center, Greenbelt, MD 20771, USA

${ }^{6}$ Yale Center for Astronomy \& Astrophysics, Physics Department, P.O. Box 208120, New Haven, CT 06520, USA

${ }^{7}$ Department of Physics, Yale University, P.O. Box 208121, New Haven, CT 06520, USA

${ }^{8}$ Jet Propulsion Laboratory, California Institute of Technology, 4800 Oak Grove Drive, Mail Stop 169-221, Pasadena, CA 91109, USA

${ }^{9}$ Departamento de Astronomía, Universidad de Chile, Santiago, Chile

${ }^{10}$ Max-Planck-Institute für extraterrestriche Physik, D-85748 Garching, Germany

${ }^{11}$ Center for Astrophysical Sciences, Department of Physics and Astronomy, Johns Hopkins University, Baltimore, MD, 21218, USA

${ }_{12}$ Department of Physics, University of Helsinki, Gustaf Hällströmin katu 2a, FI-00014 Helsinki, Finland

${ }^{13}$ University of Maryland, Baltimore County, 1000 Hilltop Circle, Baltimore, MD 21250, USA

${ }^{14}$ Department of Math \& Science, Wheelock College, 200 Riverway, Boston, MA 02215, USA

${ }^{15}$ Smithsonian Astrophysical Observatory, 60 Garden Street, Cambridge, MA 02138, USA

${ }^{16}$ Department of Physics MC 0435, Virginia Polytechnic Institute and State University, 850 West Campus Drive, Blacksburg, VA 24061, USA

${ }^{17}$ National Astronomical Observatories, Chinese Academy of Sciences, 20A Datun Road, Chaoyang District, Beijing 100012, China

${ }^{18}$ Department of Physics \& Astronomy, Clemson University, Clemson, SC 29634, USA

${ }^{19}$ Department of Physics, Drexel University, 3141 Chestnut Street, Philadelpha, PA 19104, USA

${ }^{20}$ Institute for Astronomy, Department of Physics, ETH Zurich, Wolfgang-Pauli-Strasse 27, CH-8093 Zurich, Switzerland

${ }^{21}$ Instituto de Astrofísica, Facultad de Física, Pontificia Universidad Católica de Chile, Casilla 306, Santiago 22, Chile Received 2017 June 30; revised 2017 August 18; accepted 2017 August 20; published 2017 September 26

\begin{abstract}
We present results of a ground-based near-infrared campaign with Palomar TripleSpec, Keck NIRSPEC, and Gemini GNIRS to target two samples of reddened active galactic nucleus (AGN) candidates from the $31 \mathrm{deg}^{2}$ Stripe $82 \mathrm{X}$-ray survey. One sample, which is $\sim 89 \%$ complete to $K<16$ (Vega), consists of eight confirmed AGNs, four of which were identified with our follow-up program, and is selected to have red $R-K$ colors $(>4$, Vega). The fainter sample $(K>17$, Vega) represents a pilot program to follow-up four sources from a parent sample of 34 that are not detected in the single-epoch SDSS catalog and have WISE quasar colors. All 12 sources are broad-line AGNs (at least one permitted emission line has an FWHM exceeding $1300 \mathrm{~km} \mathrm{~s}^{-1}$ ) and span a redshift range $0.59<z<2.5$. Half the $(R-K)$-selected AGNs have features in their spectra suggestive of outflows. When comparing these sources to a matched sample of blue Type 1 AGNs, we find that the reddened AGNs are more distant $(z>0.5)$, and a greater percentage have high X-ray luminosities $\left(L_{\mathrm{X}}\right.$,full $\left.>10^{44} \mathrm{erg} \mathrm{s}^{-1}\right)$. Such outflows and high luminosities may be consistent with the paradigm that reddened broad-line AGNs represent a transitory phase in AGN evolution as described by the major merger model for black hole growth. Results from our pilot program demonstrate proof of concept that our selection technique is successful in discovering reddened quasars at $z>1$ missed by optical surveys.
\end{abstract}

Key words: galaxies: active - infrared: galaxies - quasars: general - quasars: supermassive black holes X-rays: galaxies

\section{Introduction}

To understand the growth of supermassive black holes over cosmic time, it is crucial to identify and study samples of active galactic nuclei (AGNs) with diverse properties. Powerful emission from the accreting black hole imprints signatures on its surroundings that enable these sources to be detected across the electromagnetic spectrum. Optical emission from the accretion disk and gas photoionized by the AGNs can be prominent. Indeed, hundreds of thousands of AGNs have been detected by large ground-based optical surveys, like the Sloan Digital Sky Survey (York et al. 2000), with almost 300,000 Type 1 quasars in the most recent release of the SDSS Quasar Catalog (Pâris et al. 2017). These Type 1 quasars are sources where we have a direct view of the growing black holes, allowing them to be easily identified at optical wavelengths due to their blue color, which imparts a power-law slope to the optical spectra, and broad emission lines, from gas rapidly orbiting near the black hole.

However, obscured AGNs, where a direct view of the central engine is blocked by the circumnuclear torus of the AGN unification scheme (Type 2 AGNs; Antonucci 1993; Urry \& Padovani 1995) and/or large amounts of dust from the host galaxy, are less efficiently detected based on their optical emission alone. In apparent defiance of the canonical AGN unification scheme, red quasars are typically broad-line AGNs, yet are enshrouded by large amounts of dust that reddens the spectrum and attenuates optical emission. Studies of this 
extreme segment of the obscured AGN population indicate that their reddening is due to a stage of AGN evolution in the major merger model of black hole growth, rather than the orientation of the putative torus with respect to the line of sight (e.g., Georgakakis et al. 2009; Glikman et al. 2012).

According to this model (Sanders et al. 1988; Hopkins et al. 2008), when galaxies of comparable mass collide and coalesce, gas is funneled to the central supermassive black hole, which ignites AGN activity and circumnuclear star formation. During this phase, the AGN is cocooned within large amounts of dust and gas associated with ongoing star formation, potentially reaching Compton-thick levels $\left(N_{\mathrm{H}}>1.25 \times 10^{24} \mathrm{~cm}^{-2}\right.$; Kocevski et al. 2015; Ricci et al. 2017), causing the AGN to appear heavily reddened while it is intrinsically luminous. According to this major merger evolution model, powerful winds from the AGN eventually clear out the obscuring material, revealing a blue Type 1 quasar and potentially regulating galaxy growth by shutting down star formation and/or evacuating gas from the host (Hopkins et al. 2006). While the Compton-thick phase lasts $10^{7}-10^{8}$ years (Hopkins et al. 2005; Treister et al. 2010), the reddened AGN phase, when the quasar begins to clear out its surroundings, is shorter lived, $\sim 5 \times 10^{6}$ years (Hopkins et al. 2005; Glikman et al. 2012), making these sources rare. This pathway for black hole growth only pertains to a portion of the AGN population (Treister et al. 2012; Hopkins et al. 2014), with secular processes apparently responsible for triggering moderate luminosity AGNs (e.g., Kocevski et al. 2012; Schawinski et al. 2012; Villforth et al. 2014) and some high-luminosity AGNs (Villforth et al. 2017). Identifying reddened AGNs that may be in this evolutionary phase provides us with an opportunity to test whether there is a causal or coincidental connection between mergers and black hole growth (Mechtley et al. 2016; Farrah et al. 2017) and learn about how black holes can shape their environment.

Many previous red quasar samples were identified by their radio, optical-to-near-infrared (NIR), NIR, and/or mid-infrared (MIR) emission (e.g., Glikman et al. 2007, 2012, 2013; Banerji et al. 2012, 2013, 2015; Eisenhardt et al. 2012; Assef et al. 2015; Ross et al. 2015; Hamann et al. 2017). The traits of some of these sources are consistent with being in the transitory reddened phase in the AGN evolution model: they are intrinsically luminous, after correcting for extinction (Glikman et al. 2007, 2012, 2013; Assef et al. 2015; Banerji et al. 2015); they host outflows that may impart feedback onto the host galaxy (Urrutia et al. 2009; Farrah et al. 2012; Zakamska et al. 2016); and their host galaxies have morphologies consistent with having recently undergone a merger (Urrutia et al. 2008; Glikman et al. 2015). As X-ray emission provides a direct probe of black hole fueling and can pierce through optically obscuring dust, honing in on this emission provides a complementary method for identifying this population. Indeed, reddened AGNs selected from the $\sim 2 \mathrm{deg}^{2} X M M$-COSMOS survey (Hasinger et al. 2007; Cappelluti et al. 2009; Brusa et al. 2010) are launching powerful outflows, suggesting that they may be in the "clear-out" phase in the AGN evolution paradigm (Brusa et al. 2015a, 2015b, 2016; Perna et al. 2015, 2017).

Before the launch of focusing X-ray instruments with sensitivity beyond a few $\mathrm{keV}$, there had not been wide-area $\mathrm{X}$-ray surveys with sufficient depth to identify heavily obscured AGNs beyond the local universe, limiting our census of these rare, reddened sources. Stripe $82 \mathrm{X}$ is a $\sim 31 \mathrm{deg}^{2} \mathrm{X}$-ray survey with XMM-Newton and Chandra (LaMassa et al. 2013a, 2013b, 2016c; Ananna et al. 2017) that overlaps the legacy SDSS Stripe 82 field (Frieman et al. 2008). About $20 \mathrm{deg}^{2}$ of the Stripe $82 \mathrm{X}$ survey is from a dedicated XMM-Newton observing program from AO10 and AO13 (PI: Urry), reaching depths of 5-7.5 ks, while the rest of the survey is composed of archival XMM-Newton and Chandra observations in the field. The flux limit at half the survey area is $\sim 5.4 \times 10^{-15}$ $\mathrm{erg} \mathrm{s}^{-1} \mathrm{~cm}^{-2}$ in the soft band $(0.5-2 \mathrm{keV})$. Due to the relatively wide area covered in X-rays plus rich multiwavelength data, this survey provides an ideal data set to identify reddened AGNs, building on the census from smaller-area X-ray surveys and complementing samples selected at other wavelengths.

We used a combination of optical and infrared clues to identify signatures of such obscured black hole growth. The full Stripe 82 region is covered at relatively homogeneous depths in the optical by SDSS and in the NIR by the UKIRT Infrared Deep Sky Survey (UKIDSS; Lawrence et al. 2007) and the VISTA Hemisphere Survey (VHS; McMahon et al. 2013). Although, in general, reddened extragalactic sources could be dusty starbursts that may not necessarily host an accreting black hole (e.g., some (ultra)luminous infrared galaxies), X-ray emission from Stripe $82 \mathrm{X}$ sources is a clear indicator of supermassive black hole accretion: at the relatively bright X-ray flux limits of Stripe $82 \mathrm{X}$, faint X-ray emission from star formation processes in galaxies beyond the local universe are not detectable. Optical faintness in tandem with relatively brighter infrared emission is thus consistent with heavily reddened AGNs.

We present two samples of objects that we targeted for follow-up with ground-based NIR spectroscopy using Palomar TripleSpec (Herter et al. 2008), Keck NIRSPEC (McLean et al. 1998), and Gemini GNIRS (Elias et al. 2006a, 2006b). Our "bright NIR" sample $(K<16$, Vega) consists of objects selected on the basis of red $R-K$ colors, with an X-ray to optical flux $(X / O)$ cut to mitigate contamination from stars; these sources were followed up with Palomar TripleSpec. We used Keck NIRSPEC and Gemini GNIRS to follow up our "faint" sample $(K>17$, Vega), which are sources that are not detected in the single-epoch imaging of the SDSS survey, yet have WISE colors consistent with quasars. From our follow-up campaign, the bright NIR sample is nearly complete (i.e., eight of the nine sources from the parent sample have secure spectroscopic redshifts) while the faint NIR sample represents a pilot program of a larger sample. In Sections 2 and 3, we describe the target selection and follow-up observations. We discuss the results of our spectroscopic campaigns, multiwavelength properties of the samples, and insight from spectral energy distribution (SED) analysis in Section 4. In Section 5, we compare the properties of the $R-K$ sample with a matched sample of blue Type 1 AGNs and compare both samples with reddened quasars from the literature selected at other wavelengths. We assume a cosmology of $H_{0}=67.8 \mathrm{~km} \mathrm{~s}^{-1}$ $\mathrm{Mpc}^{-1}, \Omega_{M}=0.31$, and $\Omega_{\Lambda}=0.69$ (Planck Collaboration et al. 2016)

\section{Target Selection}

We focused on photometric signatures of reddening for both samples we targeted for NIR spectroscopy. Such red colors can be induced by large amounts of dust (in the host galaxy and/or circumnuclear region) or by radio synchrotron emission (Serjeant 1996). Two of our 12 sources are detected in the 
Table 1

Bright NIR Stripe $82 \mathrm{X}$ Targets: $R-K$ vs. $X / O$ Selection

\begin{tabular}{lrrrr}
\hline \hline Stripe 82X Name & \multicolumn{1}{c}{ X-ray ID } & $r(\mathrm{AB})$ & $K($ Vega $)$ & $R-K($ Vega $)$ \\
\hline S82X 013245.41-000835.5 & 4150 & 22.39 & 15.85 & 5.92 \\
S82X 024219.20+000511.9 & $618\left(108774 C^{\mathrm{b}}\right)$ & 22.32 & 15.94 & 5.78 \\
S82X 030215.39-000335.5 & 783 & 21.37 & 15.49 & 5.25 \\
S82X 030324.58-011508.3 & 855 & 21.43 & 15.89 & 0.88 \\
S82X 232801.91-002822.9 & 1859 & 21.22 & 16.00 & 0.74 \\
\hline
\end{tabular}

Notes.

${ }^{\text {a }}$ XMM-Newton record number introduced in the Stripe 82X survey (LaMassa et al. 2013a).

${ }^{\mathrm{b}}$ Source also detected by archival Chandra observations in Stripe 82 (LaMassa et al. 2013b). The Chandra Source Catalog MSID identifying number (Evans et al. 2010) for this object is noted in parentheses.

Table 2

Faint NIR Stripe 82X Targets: WISE-selected Optical Dropouts

\begin{tabular}{|c|c|c|c|c|c|c|c|c|}
\hline Stripe $82 \mathrm{X}$ Name & 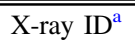 & W1 (Vega) & W2 (Vega) & W3 (Vega) & $\bar{K}(\mathrm{Vega})$ & SDSS Coadd? $^{\mathrm{b}}$ & $\overline{c r(\mathrm{AB})}$ & $\overline{R-K(\mathrm{Vega})}$ \\
\hline \multicolumn{9}{|c|}{ Keck NIRSPEC } \\
\hline S82X $022723.51+004253.3$ & $129832 \mathrm{C}$ & 16.86 & 15.84 & 12.36 & 18.75 & $\mathrm{Y}$ & 23.44 & 4.68 \\
\hline \multicolumn{9}{|c|}{ Gemini GNIRS } \\
\hline S82X 010019.25+000844.8 & $2589 \mathrm{X}$ & 16.79 & 15.28 & 12.32 & 18.36 & $\mathrm{Y}$ & 24.37 & 5.45 \\
\hline S82X 011840.06+001806.0 & $3692 X$ & 16.27 & 15.10 & 12.42 & 18.04 & $\mathrm{Y}$ & 24.40 & 5.85 \\
\hline S82X 014152.06-001749.5 & $4583 X$ & 16.14 & 14.46 & 10.87 & 17.98 & $\mathrm{~N}$ & $>24.7$ & $>6.40$ \\
\hline
\end{tabular}

Notes.

a If the X-ray ID number is followed by a "C," this indicates the Chandra MSID number from the Chandra Source Catalog (Evans et al. 2010). If the X-ray ID number is appended with an "X," this denotes the XMM-Newton record number introduced in the Stripe 82X survey (LaMassa et al. 2013a, 2016c).

${ }^{\mathrm{b}}$ Flag to indicate if optical dropout was recovered in the co-added SDSS catalog.

radio by the $1.5 \mathrm{GHz}$ FIRST survey (Helfand et al. 2015) and are discussed in more detail below. Thus, the red colors for most of these sources are likely due to obscuration.

The sources that we followed up are reported in Tables 1 and 2, where we list the full Stripe $82 \mathrm{X}$ name based on the X-ray coordinates. For clarity, we use an abbreviated version of the source name in the main text and subsequent tables.

\subsection{Bright NIR Stripe $82 X$ Sample: $R-K$ versus X/O Selection}

To unveil the brighter end of the reddened AGN population in Stripe 82X, we focus on the 551 sources (9\% of the 6181 unique X-ray sources in Stripe $82 \mathrm{X}$ ) that are detected in the $\mathrm{X}$-ray full band $^{22}$ and have UKIDSS $K$-band magnitudes brighter than 16 (Vega). We retain the sources where the SDSS $r$-band and $i$-band magnitudes are well-measured (i.e., error is below 0.5$)$ to avoid artificially reddened colors from poor photometric measurements, leaving us with 373 sources. ${ }^{23}$ For a straightforward comparison to reddened populations from other studies that use $R-K$ (Vega) to identify obscured AGNs (e.g., Banerji et al. 2012; Brusa et al. 2015a), we convert the SDSS $r$ magnitude from the $\mathrm{AB}$ system to the Bessell $R$ bandpass (Bessell 1990) in the Vega system using the formulae in Blanton \& Roweis (2007), which were calibrated on galaxies ranging in redshifts $0<z<1.5$ from the SDSS, GALEX (Martin et al. 2005), DEEP2 (Davis et al. 2003; Faber et al. 2003), and

\footnotetext{
22 The full band is defined from 0.5 to $10 \mathrm{keV}$ for XMM-Newton and from 0.5 to $7 \mathrm{keV}$ for Chandra.

${ }^{23}$ We note that only one source had $r$ - and $i$-band magnitude errors exceeding 0.5 and otherwise met our selection criteria. This source, selected as a counterpart from the SDSS co-added catalog of Jiang et al. (2014), is confused with a nearby source and a stellar spike, and hence has unreliable photometry.
}

GOODS (Giavalisco et al. 2004) surveys:

$$
\begin{gathered}
R_{\mathrm{AB}}=r-0.0576-0.3718((r-i)-0.2589), \\
R_{\mathrm{Vega}}=R_{\mathrm{AB}}-0.21,
\end{gathered}
$$

where $r$ and $i$ are the SDSS pipeline "modelMag" magnitudes, which is a PSF model for point sources, and the better of a de Vauculeurs or exponential profile fit for extended sources.

To calculate $X / O$, the ratio of $\mathrm{X}$-ray to optical flux, we use the following (see Brandt \& Hasinger 2005):

$$
X / O=\log \left(f_{\mathrm{x}} / f_{\text {opt }}\right)=\log \left(f_{\mathrm{x}}\right)+C+0.4 \times m_{r},
$$

where $C$, a constant that depends on the optical filter, is 5.67 for the SDSS $r$ band (AB; Green et al. 2004), and $m_{r}$ is the "modelMag" reported by the SDSS pipeline. Here, the X-ray flux is in the full band.

We applied a modified version of the color cuts presented in Brusa et al. (2010) to select our sample: $R-K>4$ (Vega) and $X / O>0$. We find 17 sources that meet these cuts (boxed region in Figure 1), of which seven are spectroscopically confirmed as stars and four are Type 1 AGNs with existing SDSS spectra (see Section 4.3). Of the sources lacking spectra, we removed the one object that lies along the stellar locus of the $R-K$ versus $R-W 1$ color space presented in LaMassa et al. (2016a), i.e., $R-W 1=0.998( \pm 0.02) \times(R-K)+0.18$, leaving us with five reddened AGN candidates lacking spectra (red filled squares).

We note that though the $X / O>0$ cut is designed to mitigate contamination from stars, this restriction in principle can also omit sources where the galaxy light dominates (e.g., Cardamone et al. 2007), including low-luminosity AGNs or heavily obscured to Compton-thick AGNs where the observed X-ray emission appears weak (e.g., Heckman et al. 2005; 


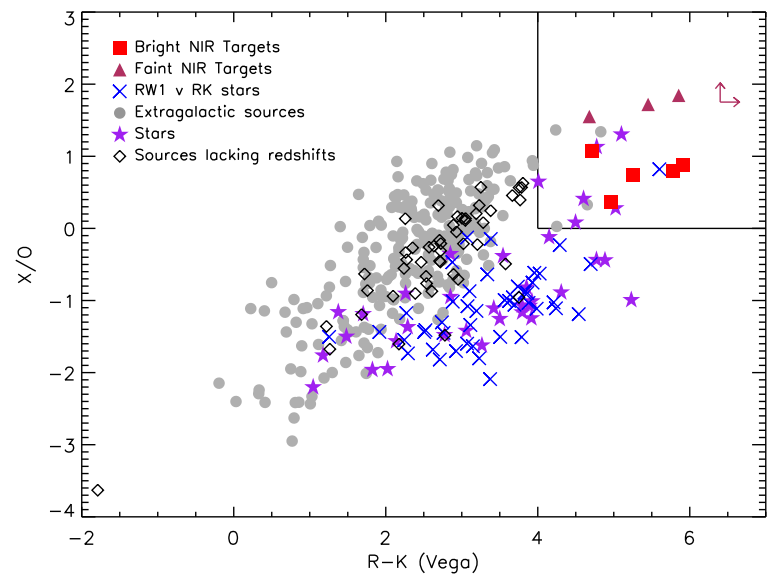

Figure 1. $R-K$ vs. $X / O$ colors of Stripe $82 \mathrm{X}$ sources brighter than $K=16$ (Vega) with significant detection in the full X-ray band (0.5-10 keV) and wellmeasured $r$ - and $i$-band magnitudes (magnitude errors less than 0.5 ). The boxed region $(R / K>4$ and $X / O>0)$ indicates the locus for reddened AGN candidates (see Brusa et al. 2010) where we defined a sample for follow-up. This sample excludes sources lacking spectra that lie along the $R-K$ vs. $R-W 1$ stellar locus presented in LaMassa et al. (2016a) since they are likely not AGNs (blue $\times$ ). The sources with pre-existing spectroscopic redshifts (from SDSS or our optical follow-up programs) that are extragalactic and stellar are shown by the gray circles and purple stars, respectively, while the sources lacking identifications via spectroscopic redshifts are indicated by the black diamonds. The reddened AGN candidates we targeted with TripleSpec on Palomar are shown by the red squares. For reference, we show where the faint NIR sample $(K>17)$ lies in this parameter space with the maroon triangles and lower limits.

LaMassa et al. 2009, 2011). The X-ray to optical flux cut selects AGNs where the X-ray emission dominates over the host galaxy, implicitly favoring AGNs with high X-ray luminosities. However, as Figure 1 shows, the sources at $R-K>4$ with $X / O<0$ are either spectroscopically confirmed as stars or are likely stars based on their $R-K$ and $R-W 1$ colors.

We targeted the five reddened AGN candidates lacking spectra with Palomar TripleSpec, as discussed below, and summarize their properties in Table 1, where the magnitudes are given in the native units from their parent catalogs, while the $R-K$ color is in the Vega system, following the derivation above. The corresponding X-ray identification numbers refer to those published in Evans et al. (2010), for Chandra sources identified in the Chandra Source Catalog, and LaMassa et al. (2013a, 2013b, 2016c), for Stripe 82X sources detected by XMM-Newton. We discuss the four extragalactic sources that have SDSS spectra in Section 4.3 and include them in our subsequent analysis.

\subsection{Faint NIR Stripe $82 X$ Sample: Optical Dropouts Recovered by WISE}

An interesting population is X-ray sources that lack an optical counterpart in the single-epoch SDSS imaging, but which are detected at infrared wavelengths. The depths of the single-epoch SDSS imaging, ${ }^{24}$ NIR imaging, ${ }^{25}$ and MIR imaging $^{26}$ in Stripe 82 are comparable for an AGN SED. $R-K \sim 3.6$ at the flux limits of these surveys, indicating that the infrared coverage is not systematically deeper than the

\footnotetext{
$24 r \leqslant 22.2$ and $i \leqslant 21.3(\mathrm{AB})$ for $95 \%$ completeness of point sources.

$25 K<18.1$ (Vega) for $5 \sigma$ point source detection.

$265 \sigma$ limit at $W 1<17.30$ and $W 2<15.84$ (Vega) for $95 \%$ sky coverage.
}

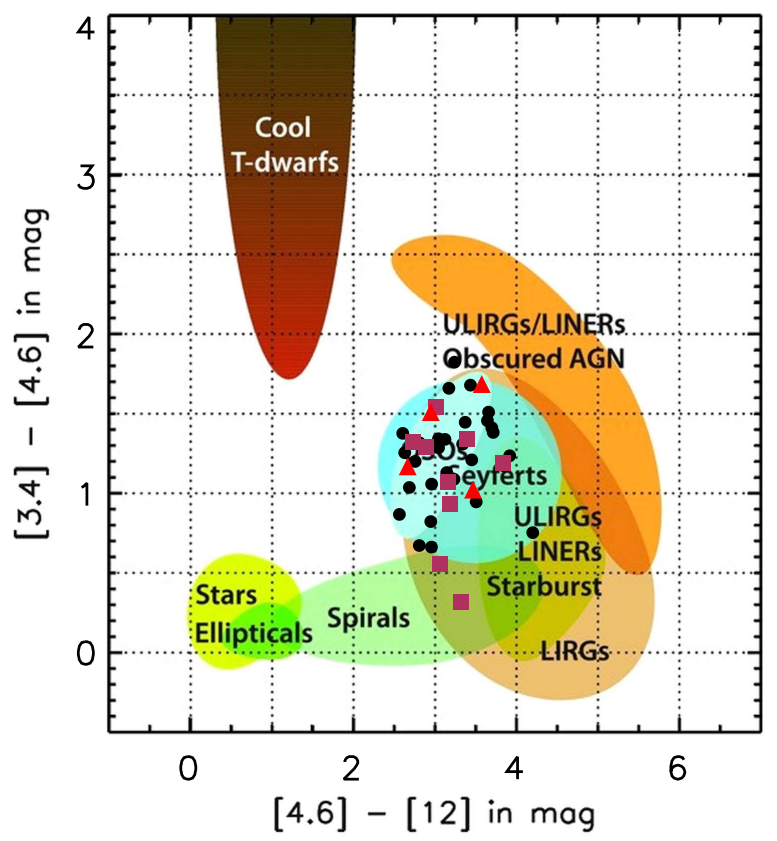

Figure 2. WISE color-color plot (Wright et al. 2010) for our parent sample of reddened AGN candidates that are optical dropouts in single-epoch SDSS imaging and have NIR detections in the least the VHS $K$ band and WISE colors in the QSO and Seyfert locus (cyan circle). The red triangles indicate the objects from our pilot program where we identified emission lines and were thus able to determine redshifts and confirm these sources as quasars; the black circles represent sources from the parent sample currently lacking identifications. We show the $R-K$ vs. $X / O$ selected sources from the bright NIR sample as maroon squares in this color space for reference.

optical. Hence, a non-detection in SDSS in conjunction with an NIR detection selects for reddening.

We create a target list of 47 such optical dropouts that have MIR colors in the quasar locus of the WISE color-color diagram (Figure 2; Wright et al. 2010) and have $K$-band detections in VHS, which is deeper than UKIDSS. These optical dropouts have no SDSS source within the nominal search radius we used to identify counterparts to the X-ray source ( $5^{\prime \prime}$ for Chandra and 7 " for XMM-Newton). We vet each potential target by eye to remove any sources that fall out of the SDSS footprint ${ }^{27}$ or where visual inspection shows a clear source that failed to be detected by the SDSS pipeline, leaving us with 37 objects.

Thirty-two of these sources are detected in the deeper coadded SDSS catalogs, while five remain undetected (Jiang et al. 2014; Fliri \& Trujillo 2016). We further vet our target list to preserve the reddening criterion of our selection using the information from the co-added SDSS catalogs. If the source is detected in the $r$ and $i$ bands in the co-added SDSS catalog such that we can calculate $R$, we only retain the sources where $R-K>4$. We also retain all sources that remain undetected in at least the $r$ or $i$ band even in this deeper imaging, which implies colors of $R-K>5.9$.

In total, we have 34 such reddened AGN candidates in our faint sample, including the five sources not detected in any SDSS band in the co-added catalogs. We targeted six with NIRSPEC on Keck (two in 2014 September and four in 2015 October; Section 3.2) and three with GNIRS on Gemini

\footnotetext{
27 Though the X-ray observations are designed to overlap the Stripe 82 region, some of the archival X-ray observations partially overlap the Stripe while the rest of the field of view is outside of the SDSS footprint.
} 
(Section 3.3). Although all sources were detected in the NIRSPEC $K$-band spectra, we were only able to identify an emission line in one object. It is likely that the remaining sources were unidentified because of the limited wavelength range in the $K$-band order. Indeed, when using the crossdispersed mode on Gemini GNIRS, which yields simultaneous $J$-, $H$-, and $K$-band coverage, we detected emission lines in all three targets.

For the remainder of this work, we focus on the four objects for which we were able to identify emission lines and thus derive redshifts. We list this sample in Table 2, where we note whether a reliable optical counterpart was found in the deeper co-added SDSS catalog, and provide the $R-K$ colors or a lower limit (if undetected in the co-added SDSS catalogs).

\section{Observations and Data Analysis}

\subsection{Palomar TripleSpec}

TripleSpec simultaneously covers wavelengths $1-2.4 \mu \mathrm{m}$ in four orders, with an approximate resolution of $120 \mathrm{~km} \mathrm{~s}^{-1}$ (based on the instrument specifications ${ }^{28}$ ). All five sources from the bright NIR Stripe $82 \mathrm{X}$ sample were observed using the standard ABBA nodding sequence, where we integrated for 300 s per exposure. Four of the sources were observed on 2015 October 27 (S82X 0242+0005, S82X 0302-0003, S82X 0303 -0015 , S82X 2328-0028) with three ABBA sequences. The fifth source, S82X 0132-0008, was observed on 2016 December 12 with four ABBA sequences. At the end of the science exposures for each target, we observed a standard A0V or A1V star for telluric correction. All sources were observed at an airmass below 1.5 .

Data were reduced with the IDL program SPEXTOOL (Cushing et al. 2004), which creates normalized flat-field images, performs a wavelength calibration based on sky lines, and extracts the spectra from each order. We note that for S82X 2338-0028, we only extract the spectrum from the B position due to a bad column affecting the emission feature in the $\mathrm{A}$ nod. Telluric correction is performed on each order with XTELLCOR (Vacca et al. 2003), after which the spectra are merged into a continuous spectrum with the SPEXTOOL XMERGERORDERS routine. Finally, the spectrum is smoothed with task XCLEANSPEC using the Savitzky-Golay routine, which preserves the average resolving power using a smoothing window that is two times the slit width.

\subsection{Keck NIRSPEC}

Keck NIRSPEC is an NIR spectrograph on Keck II, with different filter wheels limiting the wavelength range of a given spectrum to a single waveband. The approximate resolution, found from measuring resolved sky lines, is $200 \mathrm{~km} \mathrm{~s}^{-1}$. We observed S82X $0227+0042$ at an airmass of $\sim 1.1$ with the $\mathrm{K}^{\prime}$ filter $(1.950-2.295 \mu \mathrm{m})$ on 2014 September 7 with the $42 \times$ 0.'79 slit. We acquired four ABBA exposures at $600 \mathrm{~s}$ per exposure. Due to an apparent error with target acquisition or the dithering script, the source was only in the slit in the A nod. We observed the A0V standard star HD 18571.

We reduced the data with the IRAF routine WMKONSPEC, ${ }^{29}$ which corrects the distortion in the $x$ and $y$ directions

\footnotetext{
${ }^{28}$ http://www.astro.caltech.edu/palomar/observer/200inchResources/ tspecspecs.html

${ }^{29}$ http://www2.keck.hawaii.edu/inst/nirspec/wmkonspec.html
}

Table 3

Gemini GNIRS Observing Log

\begin{tabular}{llc}
\hline \hline Stripe 82X Name & $\begin{array}{l}\text { Observation Dates } \\
\text { (year month date) }\end{array}$ & $\begin{array}{c}\text { Net Exposure }^{\mathrm{a}} \\
(\mathrm{s})\end{array}$ \\
\hline S82X 0100+0008 & 2016 Jan 07 & 3600 \\
& 2016 Jan 08 & 3540 \\
S82X 0111+0018 & 2015 Dec 10 & 2400 \\
S82X 0141-0017 & 2016 Jan 02 & 6480 \\
\hline
\end{tabular}

Note.

${ }^{a}$ Net exposure time after discarding observations with poor sky conditions.

before spectral extraction. Wavelength calibration was performed using sky lines. We corrected the source spectrum for telluric features using XTELLCOR_GENERAL (Vacca et al. 2003), part of the SPEXTOOL package (Cushing et al. 2004).

\subsection{Gemini GNIRS}

Our Gemini program, GN-2015B-Q-80 (PI: LaMassa), made use of GNIRS in cross-dispersed mode, with simultaneous coverage from 0.85 to $2.5 \mu \mathrm{m}$, on Gemini North. We used a slit width of 1 !" 0 to maximize the signal throughput from our sources, with a 32 lines $/ \mathrm{mm}$ grating and short blue camera. With this instrumental set-up, the approximate resolution is $550 \mathrm{~km} \mathrm{~s}^{-1} \cdot 30$

In total, we were awarded $12 \mathrm{hr}$ of queue time in Band 3. Each target was observed for three hours, including acquisition from offset stars and standard star observations, with 24-26 ABBA exposures at $300 \mathrm{~s}$ and four ABBA exposures at $270 \mathrm{~s}$. Due to varying sky conditions from changes in cloud cover, not all science exposures were included in the analysis. Thus, we discarded observations that added more noise than signal, with the resulting net exposure times listed in Table 3 for each source.

We reduced the spectra with the XDGNIRS, pipeline which calls Gemini GNIRS IRAF routines to clean pattern noise, flat-field the data, remove spikes from the data, correct the $S$-distortion, perform the wavelength calibration based on arc lamps, and extract a spectrum from the combined A and B exposures (Cooke \& Rodgers 2005). We used XTELLCOR_GENERAL (Vacca et al. 2003) to perform the telluric correction. Spectra from separate nights were averaged, weighted by the number of exposures contributing to each spectrum.

\section{Results}

\subsection{Near-infrared Spectroscopy}

For all objects, we used photometry to flux-calibrate the spectra to obtain estimates of the emission-line fluxes: we interpolated the $K$-band filter response onto the wavelength grid of our spectra, using the filter curve from UKIDSS (Hewett et al. 2006) for the bright NIR sample (Section 2.1) and from VHS for the faint NIR sample (Section 2.2). The integrated flux is then measured from this folded spectrum. The ratio of the $K$-band flux, derived from the observed $K$-band catalog photometry, and this pseudo-flux gives us the scale factor by which we adjust the spectrum for an absolute flux calibration. We note that variability between the photometric and spectroscopic observations induce

\footnotetext{
30 https://www.gemini.edu/node $/ 1046 ? q=$ node $/ 10543$
} 

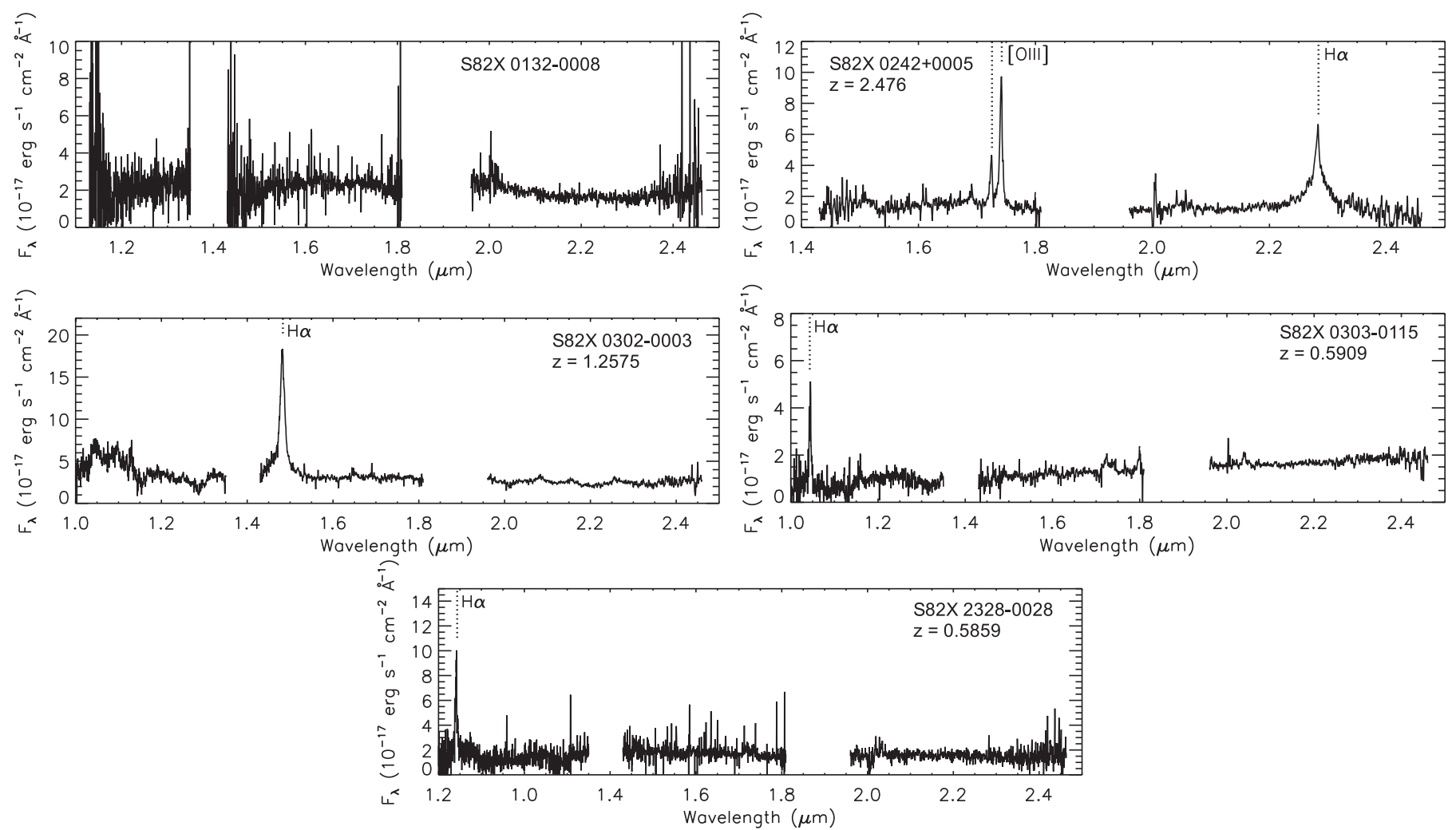

Figure 3. Palomar TripleSpec spectra of our bright NIR $R-K$ vs. $X / O$ selected sample. (Top left) Spectrum of S82X 0132-0008; although a continuum is detected, no emission lines are present. (Top right) spectrum of S82X $0242+0005$ with $\mathrm{H} \alpha$ and the [O III] doublet marked. (Middle left) spectrum of S82X 0302-0003 with $\mathrm{H} \alpha$ marked. (Middle right) extracted spectrum of S82X 0303-0115 with $\mathrm{H} \alpha$ marked. Due to a bad column that overlaps the H $\alpha$ emission feature at the A position, the spectrum was extracted from the B position only. (Bottom) extracted spectrum of S82X 2328-0028 with H $\alpha$ marked. Marked transitions indicate the emission lines visible in the two-dimensional spectral images.
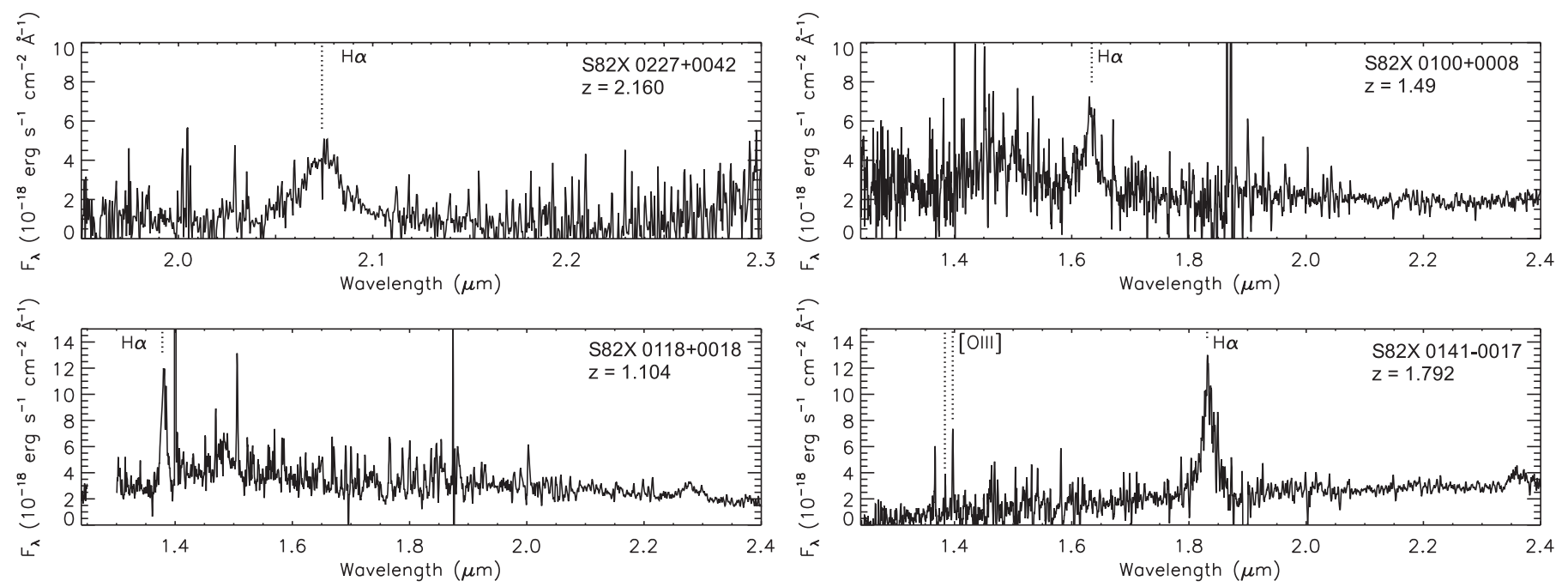

Figure 4. Spectra of our faint NIR, SDSS dropout sample. The spectrum in the top left is from Keck NIRSPEC while the others are from Gemini GNIRS. Marked transitions indicate the emission lines visible in the two-dimensional spectral images. We detected $\mathrm{H} \alpha$ emission in each source and [O III] emission in S82X $0141-0017$

uncertainty into this calibration beyond the statistical errors we report on the emission-line fluxes.

The spectra for the sources from the bright and faint samples are shown in Figures 3 and 4, respectively. In the Palomar TripleSpec spectrum of source S82X 0132-0008, we clearly detect a continuum, yet we find no emission lines, preventing us from including this source in the analysis and discussion below.
We note that the photometric redshift for this source is $z_{\text {phot }} \sim 1.74$ (Ananna et al. 2017), such that $\mathrm{H} \alpha$ would fall between the TripleSpec spectral orders, consistent with our lack of emission-line detections. For the remaining eight sources, we detected $\mathrm{H} \alpha$ emission, and in two objects (S82X 0242+0005 and S82X 0141-0017), we also detect [O III] emission. We indicate these emission features in the extracted one-dimensional 
spectra in Figure 3. We verified that these emission lines are visible in the two-dimensional spectral images.

To obtain precise redshift measurements of these sources, as well as to calculate emission-line fluxes and FWHMs of the emission lines, we analyzed the spectra in IDL. To start, we interactively fit a first-order polynomial, which was subsequently subtracted from the spectrum, to the regions of the continuum free of emission and sky lines. We then used the IDL tool MPFITFUN to fit a Gaussian model to the emission lines (Markwardt 2009). In two cases (S82X 0242+0005 and S82X 0302-0003), two broad Gaussian components were required to adequately fit the $\mathrm{H} \alpha$ emission. When fitting the [O III] doublet, the amplitude of the $4959 \AA$ line was fixed to one-third the amplitude of the $5007 \AA$ line, and the width of the lines were tied together. We note that in S82X 0242+0005, the $[\mathrm{O}$ III] doublet has a blue wing to the narrow profile, which we accommodated with additional Gaussian components; we comment more on this feature in Section 4.7. Although the redshifts were not tied when fitting the $\mathrm{H} \alpha$ and [O III] lines, we obtained consistent redshifts when fitting these features independently, indicating no systematic [O III] blueshift.

We corrected the emission-line FWHMs for the instrumental resolution using the relation $\mathrm{FWHM}_{\text {corrected }}=$ $\sqrt{\mathrm{FWHM}_{\text {observed }}^{2}-\mathrm{FWHM}_{\text {instrument }}^{2}}$ and the instrumental resolutions listed in Section 3. The emission-line fits are shown in Figures 5 and 6 and the derived redshifts and emission-line properties are listed in Tables 4 and 5, respectively. The quoted errors represent the propagation of the returned uncertainties associated with the fitted parameters.

All of the sources we observed with this program have an $\mathrm{H} \alpha$ FWHM exceeding $1300 \mathrm{~km} \mathrm{~s}^{-1}$. As pointed out by Zakamska et al. (2003), the FWHM dividing line between Type 1 and Type 2 AGNs is not firmly established. Some studies use an FWHM value of $2000 \mathrm{~km} \mathrm{~s}^{-1}$ to differentiate between Type 1 and Type 2 AGNs (e.g., Zakamska et al. 2003; Alexandroff et al. 2013), while others set the limit at $1000 \mathrm{~km} \mathrm{~s}^{-1}$ (e.g., Weedman 1977) or $1100 \mathrm{~km} \mathrm{~s}^{-1}$ (Reyes et al. 2008). Hao et al. (2005) demonstrated that the distribution of $\mathrm{H} \alpha$ FWHMs for emission-line galaxies in SDSS is bimodal: all broad-line sources have a minimum FWHM of $1200 \mathrm{~km} \mathrm{~s}^{-1}$. They thus define any source with an FWHM above this value as a Type 1 AGN. Following this convention, and for consistency with previous red quasar studies that require an FWHM exceeding $1000 \mathrm{~km} \mathrm{~s}^{-1}$ to define a source as a quasar (Glikman et al. 2007), we classify all of our Stripe 82X sources as Type 1 AGNs. We note, however, that the classification of the two sources with $\mathrm{H} \alpha$ FWHMs below $2000 \mathrm{~km} \mathrm{~s}^{-1}$, S82X 0303-0115 and S82X 2328-0028, may be ambiguous. As discussed below, all have X-ray luminosities consistent with accretion onto a supermassive black hole.

\subsection{X-Ray Properties of AGNs Targeted with Infrared Spectroscopy}

From the redshifts measured above, we calculated the $k$-corrected, observed (non-absorption corrected) full-band Xray luminosities $\left(L_{\mathrm{X} \text {,full }}\right.$, where $f_{k \text {-corr }}=f_{\text {observed }} \times(1+z)^{\Gamma-2}$ and $\Gamma$, the slope of the AGN continuum power law, is 1.7; LaMassa et al. 2013a, 2013b, 2016c). To estimate the approximate X-ray absorption, we calculated the hardness ratio: $H R=(H-S) /(H+S)$, where $H$ is the net number of counts in the hard band and $S$ is the net number of counts in the soft band. We note that while the soft range is $0.5-2 \mathrm{keV}$ for both Chandra and XMM-Newton, the hard band is $2-10 \mathrm{keV}$ for XMM-Newton and $2-7 \mathrm{keV}$ for Chandra. For this calculation, we used the Bayesian Estimation of Hardness Ratios (BEHR; Park et al. 2006), which provides robust estimates of $H R$ in the low-count regime and in the case of nondetections in either band. BEHR takes as input the total counts in the soft and hard bands within user-defined source and background regions and the ratio of the areas between the source and background regions. While XMM-Newton has three detectors, we extracted the net counts from only the MOS1 detector for a straightforward comparison with model $H R$ values.

Gas column density estimates $\left(N_{\mathrm{H}}\right)$ derived from hardness ratios are redshift dependent: at higher redshifts, the higherenergy photons $(>7-10 \mathrm{keV})$, which are less attenuated by absorption, are shifted into the observed bandpass. Assuming an absorbed power law, we calculated a grid of hardness ratios for various $N_{\mathrm{H}}$ values over a range of redshifts in bins of 0.05 for both Chandra and the MOS1 detector on XMM-Newton. Using the redshift of the source and range of hardness ratios returned by BEHR, we determined the implied $N_{\mathrm{H}}$ and report these values in Table 4. Of the eight sources, the hardness ratios for three are consistent with an unabsorbed X-ray source and three have lower limits on $N_{\mathrm{H}}$ of 0 ; we do not correct for Galactic absorption since such low column densities $\left(N_{\mathrm{H}} \sim 3 \times 10^{20} \mathrm{~cm}^{-2}\right.$; Dickey \& Lockman 1990) have little impact on the X-ray spectrum.

Since S82X $0242+0005$ is detected in hard X-rays $\left(2-10 \mathrm{keV} ; \quad F_{2-10 \mathrm{keV}}=(1.56 \pm 0.34) \times 10^{-14} \mathrm{erg} \mathrm{s}^{-1}\right)$ and the [O III] $5007 \AA$ line is measured, we can independently assess the X-ray obscuration by $F_{2-10 \mathrm{keV}} / F_{\mathrm{O} \text { III }}$ : because [O III] forms in the AGN narrow-line region, it is unaffected by the circumnuclear obscuration that attenuates the $\mathrm{X}$-ray emission and thus serves as a proxy of the intrinsic AGN luminosity (e.g., Bassani et al. 1999; Heckman et al. 2004; LaMassa et al. 2010). The ratio of the hard X-ray to [O III] flux can then indicate whether the X-ray emission is heavily absorbed (Bassani et al. 1999; Panessa et al. 2006; Lansbury et al. $2014,2015)$. We find $\log \left(F_{2-10 \mathrm{keV}} / F_{\mathrm{O} \text { III }}\right)=0.72 \pm 0.09$ dex, which is significantly less than the mean value for unabsorbed Type 1 AGNs (1.59 \pm 0.48 dex Heckman et al. 2005), but higher than the most heavily obscured, Compton-thick systems (e.g., LaMassa et al. 2009, 2011). Both $F_{2-10 \mathrm{keV}} / F_{\mathrm{O} \text { III }}$ and the implied $N_{\mathrm{H}}$ from the hardness ratio are consistent with a moderately obscured AGN. However, the narrow-line region can suffer extinction, which would translate into more luminous intrinsic [O III] emission, causing the true $F_{2-10 \mathrm{keV}} / F_{\mathrm{O} \text { III }}$ to decline. The implied X-ray column density can thus be higher.

We caution that the theoretical $H R-N_{\mathrm{H}}$ conversion assumes a simple absorbed power law while the observed X-ray spectra of obscured AGN are generally more complex, with scattered emission or leakage through a patchy obscuring medium that can boost the observed soft X-ray flux compared with the model assumed here. Furthermore, hardness ratios provide no information about the global distribution, or global column density, of obscuration around the AGN. Indeed, AGNs can have significantly different line-of-sight and global column densities (LaMassa et al. 2014; Yaqoob et al. 2015; LaMassa et al. 2016b). Though the implied column densities for some of these AGNs are consistent with the column densities of FIRST2MASS-selected reddened quasars (Glikman et al. 2012) 

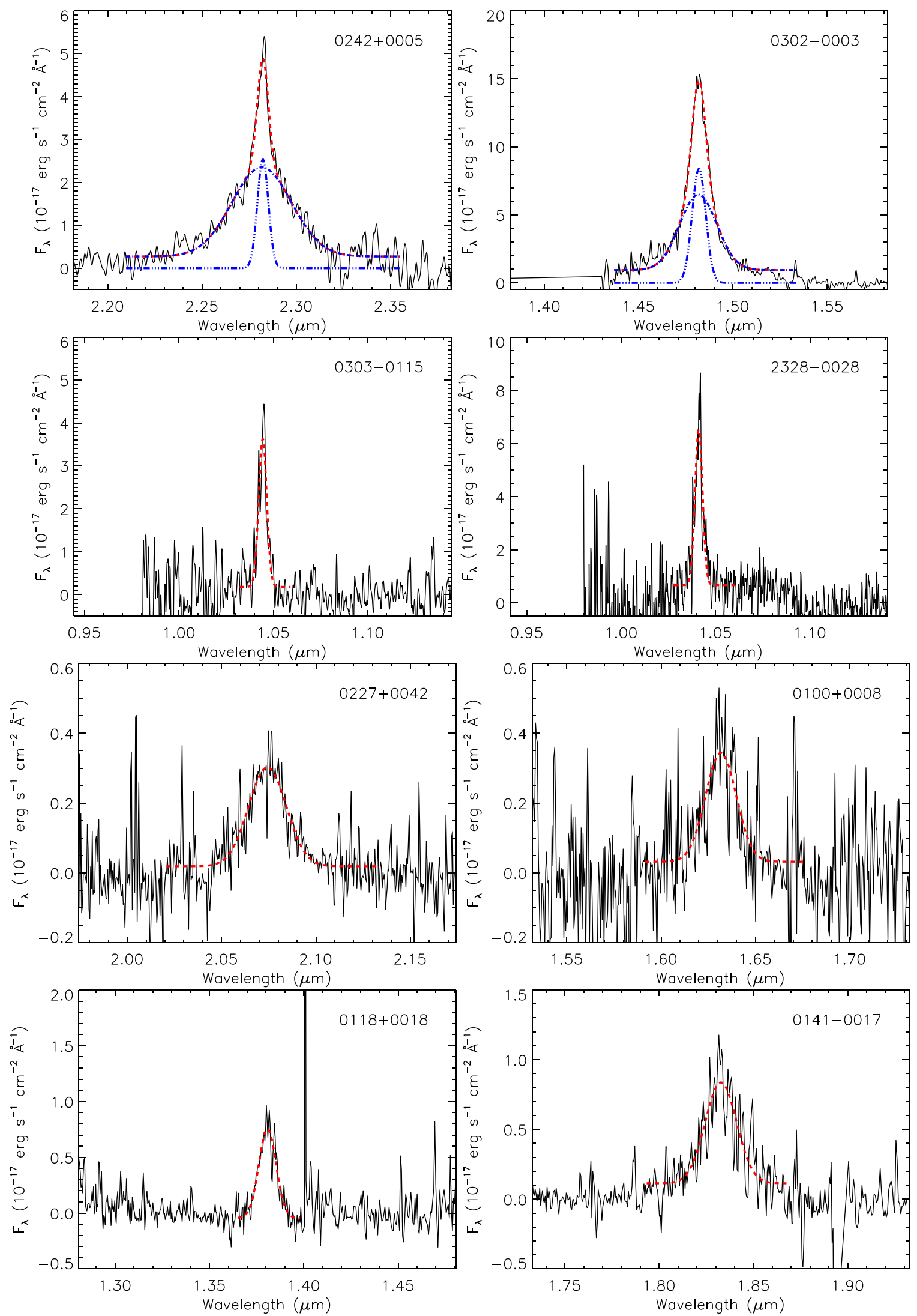

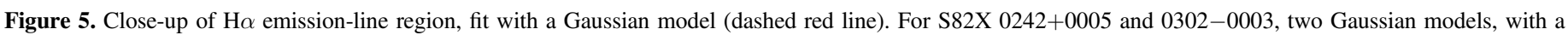

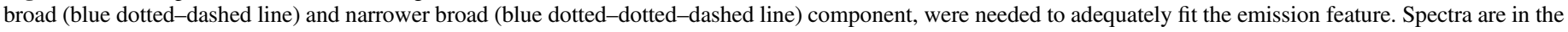
observed frame. 

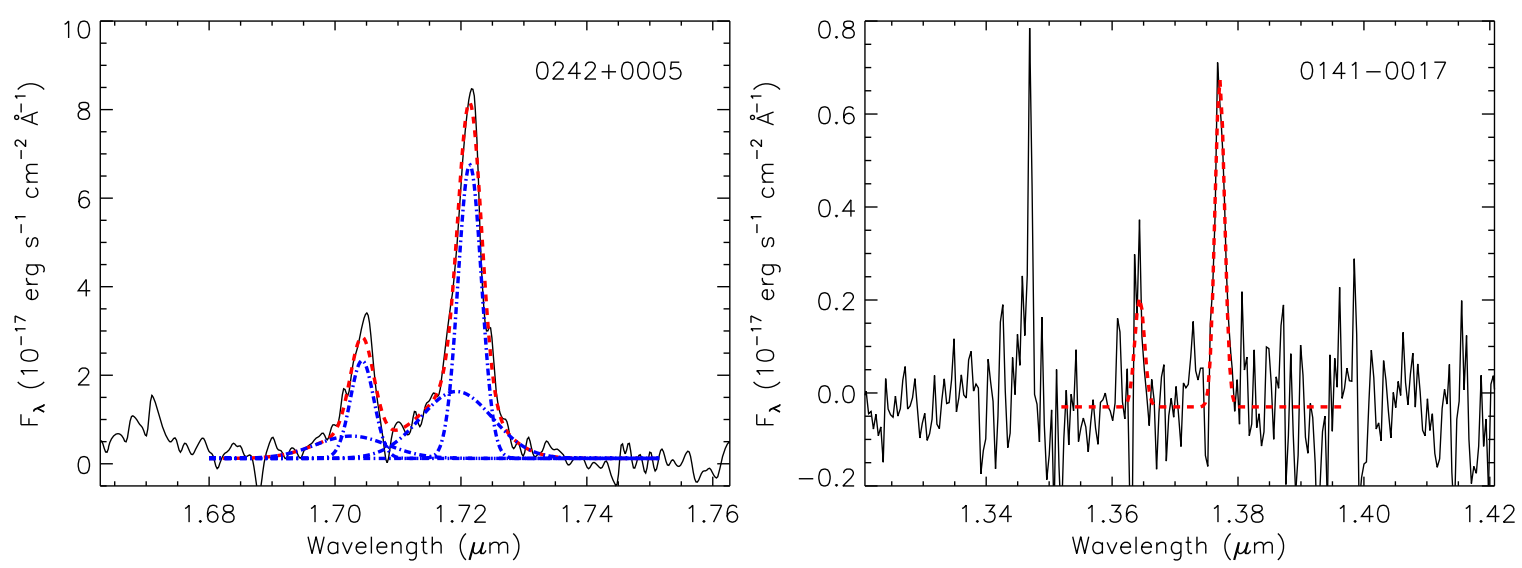

Figure 6. Close-up of the [O III] doublet fitted with a two-component Gaussian model, with the width of the lines tied together and the amplitude of the $4959 \AA$ line frozen to a third of the $5007 \AA$ line. We included an additional Gaussian component to fit the blue wing to the [O III] doublet in S82X $0242+0005$ (left), which is blueshifted with respect to the narrow component by $\Delta v=-400 \mathrm{~km} \mathrm{~s}^{-1}$, suggestive of outflowing gas. The red dashed line indicates the sum of the emission lines and the blue dotted-dashed line represents the individual narrow and broad components. Spectra are in the observed frame.

derived via X-ray spectral fitting (LaMassa et al. 2016b), the reported $N_{\mathrm{H}}$ ranges should be considered approximate.

\subsection{Bright Sources with SDSS Spectra}

Four Stripe 82X sources with $R-K>4$ and $X / O>0$ have existing SDSS spectra and obey the quality control cuts and magnitude limits applied to our target list for the bright NIR sample. The optical, infrared, and X-ray properties of these sources are presented in Table 6 . As can be seen by their SDSS spectra in Figure 7, they are all Type 1 AGNs.

We also calculated BEHR-derived hardness ratios for these sources to estimate their column densities. While one source only has an upper limit on the implied column density, the other three objects have hardness ratios consistent with non-zero absorption. Two of these sources, S82X $0022+0020$ and S82X 0040+0058, are detected in hard X-rays and are at sufficiently low redshift that [O III] $5007 \AA$ is observed in the SDSS spectrum. Based on our fits to the optical spectra (see Section 4.6.2), we find rest-frame [O III] $5007 \AA$ flux values of $(2.06 \pm 0.17) \times 10^{-15} \mathrm{erg} \mathrm{s}^{-1} \mathrm{~cm}^{-2}$ and $(3.2 \pm 0.2) \times 10^{-16} \mathrm{erg} \mathrm{s}^{-1} \mathrm{~cm}^{-2}$ for S82X $0022+0020$ and S82X 0040+0058, respectively. With observed hard X-ray fluxes of $(1.7 \pm 0.2) \times 10^{-14} \mathrm{erg} \mathrm{s}^{-1} \mathrm{~cm}^{-2}$ and $(1.5 \pm 0.2) \times$ $10^{-13} \mathrm{erg} \mathrm{s}^{-1} \mathrm{~cm}^{-2}$, we obtain $\log \left(F_{2-10 \mathrm{keV}} / F_{\mathrm{O} \text { III }}\right)$ values of $0.92 \pm 0.06 \mathrm{dex}$ and $2.68 \pm 0.07 \mathrm{dex}$ for $\mathrm{S} 82 \mathrm{X} 0022+0020$ and S82X 0040+0058. These values are consistent with the hardness ratios: S82X 0022+0020 is moderately X-ray obscured while S82X $0040+0058$ is X-ray unobscured.

Although this SDSS sample is optically brighter than the sources we identified with our spectroscopic campaign, their $\mathrm{X}$-ray luminosities span a similar range. Below, we consider these four sources alongside the four we targeted with Palomar TripleSpec when we discuss the bright NIR $R-K$ versus $X / O$ Stripe $82 X$ sample.

\subsection{Radio Properties}

As noted above, red colors can be induced by synchrotron emission from jets along the line of sight that boost the $K$-band flux (e.g., Serjeant 1996). The FIRST survey covers the full Stripe 82 region (Helfand et al. 2015), and only 2 of our 12 sources are detected by FIRST: S82X 0011+0057 and S82X 0302-0003. Following the prescription to calculate radio loudness from Ivezić et al. (2002), we first define an AB radio magnitude based on the integrated FIRST flux density at $20 \mathrm{~cm}$ :

$$
t=-2.5 \log \left(\frac{F_{\text {int }}}{3631 \mathrm{Jy}}\right) .
$$

The FIRST flux densities are $156 \mathrm{mJy}$ and $0.46 \mathrm{mJy}$ for S82X 0011+0057 and S82X 0302-0003, respectively, corresponding to $t=10.9$ and 17.2. We then calculate the radio loudness by taking the ratio of the radio and optical flux:

$$
R=\log \left(\frac{F_{\text {radio }}}{F_{\text {optical }}}\right)=0.4\left(m_{r}-t\right),
$$

where $m_{r}$ is the SDSS $r$-band magnitude. We note that the $r$ band magnitude is not corrected for extinction, making our $R$ values upper limits. We find $R=3.9$ and 1.7 for S82X 0011 +0057 and S82X 0302-0003, respectively. While S82X 0302 -0003 can be classified as radio intermediate $(1<R<2$; Miller et al. 2011), S82X $0011+0057$ is radio loud. The prominent radio jet in this source might contribute to the $K$-band flux, which may enhance the red $R-K$ color.

\subsection{SED Analysis}

Using any available photometric data from ultraviolet to MIR wavelengths, we constructed the SEDs of these sources (Ananna et al. 2017). The ultraviolet data are from the GALEX Medium Imaging Survey (Morrissey et al. 2007). Due to the optical faintness of our sources, only one object, S82X 0011 +0057 , is detected by GALEX, and only in the near-ultraviolet band. The optical data were culled from the co-added Stripe 82 catalog from Fliri \& Trujillo (2016), if available, otherwise, they were culled from from Jiang et al. (2014); one source, S82X 0141+0017, was not detected at any optical wavelength. For the NIR data, we used VHS magnitudes where available, or UKIDSS for filters that did not have a detection in VHS. ${ }^{31}$ The

\footnotetext{
${ }^{31}$ We only used both VHS and UKIDSS magnitudes if the magnitudes in the filters in common between both observatories were consistent. For S82X 0303 -0015 , the lack of common filters between VHS and UKIDSS is due to noncoverage in the VHS $K$-band and UKIDSS $J$-band; the source was at the edge of the detector in the $J$-band in UKIDSS, precluding the UKIDSS pipeline from measuring a $J$-band magnitude. Here, we combined the UKIDSS and VHS magnitudes.
} 
Table 4

Redshifts and X-ray Characteristics of Reddened AGNs

\begin{tabular}{|c|c|c|c|c|}
\hline Source & $z$ & $\begin{array}{c}L_{\mathrm{X}, \text { full }^{\mathrm{a}}} \\
\left(\mathrm{erg} \mathrm{s}^{-1}\right)\end{array}$ & $H R^{\mathrm{b}}$ & $\begin{array}{c}N_{\mathrm{H}}{ }^{\mathrm{c}} \\
\left(10^{22} \mathrm{~cm}^{-2}\right)\end{array}$ \\
\hline \multicolumn{5}{|c|}{ Palomar TripleSpec } \\
\hline S82X 0242+0005 & $2.476 \pm 0.001$ & $5.57 \times 10^{44}$ & $-0.24_{-0.19}^{+0.25}$ & $4_{-4}^{+16}$ \\
\hline S82X 0302-0003 & $1.2574 \pm 0.0001$ & $2.51 \times 10^{44}$ & $0.37_{-0.26}^{+0.30}$ & $10_{-4}^{+10}$ \\
\hline S82X 0303-0115 & $0.5909 \pm 0.0003$ & $1.71 \times 10^{43}$ & $0.21_{-0.34}^{+0.36}$ & $3_{-2}^{+4}$ \\
\hline S82X 2328-0028 & $0.5859 \pm 0.0001$ & $1.08 \times 10^{44}$ & $-0.96_{-0.04}^{+0.04}$ & 0 \\
\hline \multicolumn{5}{|c|}{ Keck NIRSPEC } \\
\hline S82X 0227+0042 & $2.16 \pm 0.01$ & $8.17 \times 10^{44}$ & $-0.90_{-0.10}^{+0.10}$ & 0 \\
\hline \multicolumn{5}{|c|}{ Gemini GNIRS } \\
\hline S82X 0100+0008 & $1.49 \pm 0.01$ & $2.25 \times 10^{44}$ & $-0.75_{-0.25}^{+0.12}$ & 0 \\
\hline S82X 0118+0018 & $1.103 \pm 0.003$ & $1.46 \times 10^{44}$ & $-0.49_{-0.51}^{+0.17}$ & $<0.6$ \\
\hline S82X 0141-0017 & $1.792 \pm 0.004$ & $2.68 \times 10^{44}$ & $-0.43_{-0.22}^{+0.25}$ & $<3$ \\
\hline
\end{tabular}

Notes.

${ }^{a} k$-corrected (i.e., rest-frame), non-absorption-corrected luminosities.

b $H R=(H-S) /(H+S)$, where $H(S)$ are the net counts in the hard (soft) X-ray bands. Hardness ratios were calculated with BEHR (Park et al. 2006).

${ }^{\mathrm{c}}$ Gas column density $\left(N_{\mathrm{H}}\right)$ implied by the $H R$ ranges.

optical and NIR magnitudes were corrected for Galactic extinction.

In the case of non-detections, we use the upper limits reported in the various multiwavelength catalogs: $m_{\mathrm{FUV}, \mathrm{AB}}=22.6$ and $m_{\mathrm{NUV}, \mathrm{AB}}=22.7$ (GALEX $5 \sigma$ limit; Morrissey et al. 2007); $m_{\mathrm{u}, \mathrm{AB}}=24.2, m_{\mathrm{g}, \mathrm{AB}}=25.2, m_{\mathrm{r}, \mathrm{AB}}=24.7, m_{\mathrm{i}, \mathrm{AB}}=24.3$, and $m_{\mathrm{z}, \mathrm{AB}}=23.0(3 \sigma$ level for $50 \%$ completeness in the SDSS co-added catalog; Fliri \& Trujillo 2016); $\quad m_{\mathrm{J}, \mathrm{AB}}=21.5$, $m_{\mathrm{H}, \mathrm{AB}}=21.2$, and $m_{\mathrm{K}, \mathrm{AB}}=20.4(5 \sigma$ detection limit for point sources; McMahon et al. 2013); and $F_{\nu, W 1}=0.08 \mathrm{mJy}$, $F_{\nu, W 2}=0.11 \mathrm{mJy}, F_{\nu, W 3}=1 \mathrm{mJy}$, and $F_{\nu, W 4}=6 \mathrm{mJy}(5 \sigma$ limit for point sources; Wright et al. 2010).

We fit the SEDs with AGNFitter (Calistro Rivera et al. 2016) to estimate the bolometric AGN luminosities $\left(L_{\text {bol }}\right)$ and reddening $(E(B-V))$. This algorithm employs a Bayesian Markov Chain Monte Carlo (MCMC) method, assuming a flat prior on the parameters listed in Table 1 of Calistro Rivera et al. (2016), and fits the following templates to the SED: accretion disk emission, which is a modified version of the Richards et al. (2006) template, extended to wavelengths redward of $1 \mu \mathrm{m}$ assuming $F_{\nu} \propto \nu^{-2}$; hot dust emission from the putative torus using models from Silva et al. (2004); host galaxy emission using the stellar population models from Bruzual \& Charlot (2003); and cold dust emission associated with star formation using templates from Chary \& Elbaz (2001) and Dale \& Helou (2002). AGNFitter accounts for upper limits by creating a fictitious data point at half the value of the upper limit $\left(F_{\mathrm{UL}}\right)$, with an error bar of $\pm 0.5 F_{\mathrm{UL}}$, such that the upper limit data point spans the range from 0 to $F_{\mathrm{UL}}$. Inclusion of upper limits allows the MCMC sampling to accept models that lie within the bounds defined by the upper limits.

The fitted parameter space is 10 dimensional (see Calistro Rivera et al. 2016 for details), which is on the order of or larger than the number of photometric detections used in the fitting, so we caution that our results from this exercise are approximate. However, it is the best we are able to do with our data and does provide a sense of the bolometric AGN luminosity and reddening.
We ran AGNFitter with two burn-in sets, with 4000 steps per set and 100 chains per set: after each burn-in, the starting point in the parameter space of the subsequent MCMC chains is that of the highest likelihood of the previous chains. After the burnin sets, the MCMC chain is run with 10,000 steps, where all sampled areas of the parameter space are used in the calculation of the posterior probability distribution functions (PDFs).

We show the fitted SEDs in Figures 8-10, where the black circles represent our photometric data points. Ten random realizations from the MCMC chain are plotted. The sum of the individual templates from these realizations are shown by the solid red line, with the individual templates denoted by the other colored lines as indicated in the caption of Figure 8. In Table 7, we list results from the SED fitting: the AGN and host galaxy reddening values, $E(B-V)_{\mathrm{AGN}}$ and $E(B-V)_{\mathrm{Galaxy}}$, respectively; and $L_{\mathrm{bol}}$, found by integrating the luminosity from the de-reddened accretion disk template from 0.03 to $1 \mu \mathrm{m}$. We note that since AGNFitter does not include the X-ray emission when modeling the SEDs, the AGN bolometric luminosity derived from the accretion disk template is underestimated (but see Krawczyk et al. 2013 for a discussion on how the geometry of the X-ray-emitting corona determines whether the inclusion of $>2 \mathrm{keV}$ X-ray emission in the AGN bolometric luminosity amounts to "double counting" when calculating the intrinsic AGN luminosity).

To estimate black hole masses (see below), we calculate monochromatic luminosities at $5100 \AA\left(\lambda L_{5100}\right)$ and $3000 \AA$ $\left(\lambda L_{3000}\right)$ from $L_{\text {bol }}$ assuming a bolometric correction of $8.1 \pm 0.4$ and $5.2 \pm 0.2$, respectively (Runnoe et al. 2012), and list these values, where appropriate, in Table 7. The reported values derived from the SED fitting represent the median of the PDFs, with the lower and higher error bars indicating the bounds for the 16th and 84th percentiles of the PDFs.

\subsection{Black Hole Masses and Eddington Ratios}

We emphasize that due to the limited number of photometric detections used in the SED decomposition, the monochromatic 
Table 5

Emission-line Properties of Targeted Reddened AGNs ${ }^{\mathrm{a}}$

\begin{tabular}{|c|c|c|c|c|c|c|}
\hline Source & $\begin{array}{c}f_{\mathrm{H} \alpha, 1} \\
\left(10^{-17} \mathrm{erg} \mathrm{s}^{-1} \mathrm{~cm}^{-2}\right)\end{array}$ & $\begin{array}{l}\mathrm{FWHM}_{\mathrm{H} \alpha, 1} \\
\left(\mathrm{~km} \mathrm{~s}^{-1}\right)\end{array}$ & $\begin{array}{c}f_{\mathrm{H} \alpha, 2} \\
\left(10^{-17} \mathrm{erg} \mathrm{s}^{-1} \mathrm{~cm}^{-2}\right)\end{array}$ & $\begin{array}{c}\mathrm{FWHM}_{\mathrm{H} \alpha, 2} \\
\left(\mathrm{~km} \mathrm{~s}^{-1}\right)\end{array}$ & $\begin{array}{c}f_{[\mathrm{O} \text { III] }} 5007 \AA \\
\left(10^{-17} \mathrm{erg} \mathrm{s}^{-1} \mathrm{~cm}^{-2}\right)\end{array}$ & $\begin{array}{c}\mathrm{FWHM}_{[\mathrm{O} \text { III] }]} 5007 \AA \\
\left(\mathrm{km} \mathrm{s}^{-1}\right)\end{array}$ \\
\hline \multicolumn{7}{|c|}{ Palomar TripleSpec } \\
\hline S82X 0302-0003 & $1370 \pm 60$ & $4690 \pm 60$ & $760 \pm 30$ & $1720 \pm 20$ & $\cdots$ & $\cdots$ \\
\hline S82X 0303-0115 & $180 \pm 10$ & $1430 \pm 60$ & $\cdots$ & $\cdots$ & $\cdots$ & $\cdots$ \\
\hline S82X 2328-0028 & $290 \pm 10$ & $1350 \pm 20$ & $\cdots$ & $\cdots$ & $\cdots$ & $\cdots$ \\
\hline S82X 0227+0042 & $70 \pm 60$ & $3400 \pm 1300$ & $\cdots$ & $\cdots$ & $\cdots$ & $\cdots$ \\
\hline \multicolumn{7}{|c|}{ Gemini GNIRS } \\
\hline S82X 0100+0008 & $60 \pm 50$ & $3500 \pm 1200$ & $\ldots$ & $\ldots$ & $\ldots$ & $\ldots$ \\
\hline S82X $0118+0018$ & $90 \pm 40$ & $2300 \pm 300$ & $\ldots$ & $\cdots$ & $\ldots$ & $\ldots$ \\
\hline S82X 0141-0017 & $150 \pm 60$ & $3200 \pm 600$ & $\cdots$ & $\cdots$ & $<39^{\mathrm{b}}$ & $400 \pm 200$ \\
\hline
\end{tabular}

Notes.

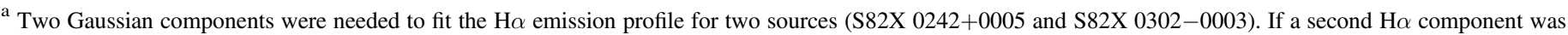

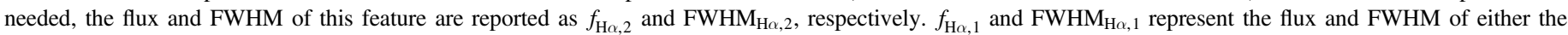

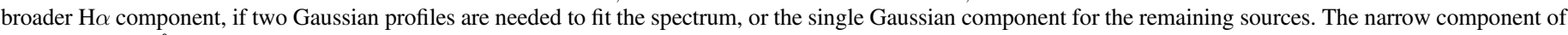
the [O III] $5007 \AA$ line is reported for S82X 0242+0005; the broad wing to the [O III] doublet is recorded in Table 8 and discussed in Section 4.7 .

b $3 \sigma$ upper limit.

and bolometric luminosities are uncertain, which propagate to uncertainties in the estimated black hole masses $\left(M_{\mathrm{BH}}\right)$ and implied accretion rate as measured by the Eddington parameter $\left(\lambda_{\text {Edd }}=L_{\mathrm{bol}} / L_{\mathrm{Edd}}\right)$. We estimate $M_{\mathrm{BH}}$ and $\lambda_{\text {Edd }}$ using the best available data, but caution that these values should be considered approximate.

\subsubsection{Targeted Sources}

For the sources that we targeted with Palomar, Keck, and Gemini, we use the estimated de-reddened $\lambda L_{5100}$ and measured $\mathrm{H} \alpha$ FWHM values to derive $M_{\mathrm{BH}}$ using

$$
\begin{aligned}
M_{\mathrm{BH}}= & (9.7 \pm 0.5) \times 10^{6}\left[\frac{\mathrm{FWHM}(\mathrm{H} \alpha)}{1000 \mathrm{~km} \mathrm{~s}^{-1}}\right]^{2.06 \pm 0.06} \\
& \times\left[\frac{\lambda L_{5100}}{10^{44} \mathrm{erg} \mathrm{s}^{-1}}\right]^{0.519 \pm 0.07} M_{\odot}
\end{aligned}
$$

from Greene et al. (2010). For the two sources where two Gaussian components were needed to fit the $\mathrm{H} \alpha$ profile ( $\mathrm{S} 82 \mathrm{X}$ 0242+0005 and S82X 0302-0003), we use the broader $\mathrm{H} \alpha$ FWHM to estimate $M_{\mathrm{BH}}$ since this component arises from gas closer to the black hole.

From these $M_{\mathrm{BH}}$ values, we estimate the Eddington luminosity $\left(L_{\mathrm{Edd}}=1.3 \times 10^{38} M_{\mathrm{BH}} / M_{\odot} \mathrm{erg} \mathrm{s}^{-1}\right.$; Frank et al. 2002) and $\lambda_{\text {Edd }}$. These values are listed in Table 7 . The errors represent the propagation of the statistical measurement errors of the individual parameters and the errors associated with the bolometric corrections and virial $M_{\mathrm{BH}}$ relations. We note that comparisons of black hole masses derived via single-epoch measurements, as calculated here, with those determined from reverberation mapping studies show a sample dispersion of $\sim 0.5$ dex (e.g., Vestergaard \& Peterson 2006), which is an additional uncertainty to our $M_{\mathrm{BH}}$ values beyond the formal errors that we report.

We caution that the view to the broad-line region is likely obscured, such that we are not getting an unbiased view of the gas kinematics near the black hole. If we are indeed viewing just the outer photosphere of the broad-line region, the FWHMs of $\mathrm{H} \alpha$ and the other broad emission lines we use below to calculate $M_{\mathrm{BH}}$ are systematically lower than those observed in unobscured AGNs that were used to derive virial relations to calculate $M_{\mathrm{BH}}$. Our estimated black hole masses may thus be lower limits to the true value.

\subsubsection{SDSS Sample}

Since $\mathrm{H} \alpha$ is not covered in the optical spectra of the sources in the bright NIR $R-K$ SDSS sample, we estimate the black hole masses, and the associated Eddington ratios, using the $\mathrm{H} \beta$ or Mg II emission lines. Two of these sources have published black hole masses in the Shen et al. (2011) catalog (S82X 0011 +0057 and S82X 0043+0052), which were calculated using the FWHM of the $\mathrm{Mg}$ II line and $\lambda L_{3000}$. For consistency with our targeted sample, we use the $\lambda L_{3000}$ we calculated from our SED fitting $\left(\lambda L_{3000}=L_{\mathrm{Bol}} /(5.2 \pm 0.2)\right)$ along with the reported Mg II FWHM in Shen et al. (2011) to estimate $M_{\mathrm{BH}}$ given the relation published in Trakhtenbrot \& Netzer (2012):

$$
\begin{aligned}
M_{\mathrm{BH}}= & 5.6 \times 10^{6}\left[\frac{\mathrm{FWHM}(\mathrm{Mg} \mathrm{II})}{1000 \mathrm{~km} \mathrm{~s}^{-1}}\right]^{2} \\
& \times\left[\frac{\lambda L_{3000}}{10^{44} \mathrm{erg} \mathrm{s}^{-1}}\right]^{0.62} M_{\odot} .
\end{aligned}
$$

The black hole masses and Eddington ratios are reported in Table 7.

For the remaining two sources, we fitted the SDSS spectra using the IRAF package SPECFIT (Kriss 1994) to obtain emission-line FWHMs. This routine uses a $\chi^{2}$ minimization technique to find the best fit to the input model parameters, which consists of (1) the AGN power-law continuum, (2) star formation templates that span an age range from $56 \mathrm{Myr}$ to 10 Gyr (S. Charlot \& G. Bruzual 2017, private communication), (3) a Cardelli et al. (1989) dust extinction that attenuates the 
Table 6

Bright NIR $R-K$ vs. $X / O$ Sample from SDSS

\begin{tabular}{|c|c|c|c|c|c|c|c|c|c|}
\hline Stripe $82 \mathrm{X}$ Name & $\mathrm{X}$-ray $\mathrm{ID}^{\mathrm{a}}$ & $\begin{array}{c}r \\
(\mathrm{AB})\end{array}$ & $\begin{array}{c}K \\
\text { (Vega) }\end{array}$ & $\begin{array}{l}R-K \\
(\text { Vega) }\end{array}$ & $X / O$ & $z$ & $\begin{array}{c}L_{\mathrm{X}, \text { full }^{\mathrm{b}}} \\
\left(\mathrm{erg} \mathrm{s}^{-1}\right)\end{array}$ & $H R^{\mathrm{c}}$ & $\begin{array}{c}N_{\mathrm{H}}^{\mathrm{d}} \\
\left(10^{22} \mathrm{~cm}^{-2}\right)\end{array}$ \\
\hline S82X 001130.21+005751.5 & $111 \mathrm{X}$ & 20.65 & 15.56 & 4.83 & 1.34 & 1.491 & $2.87 \times 10^{45}$ & $-0.30_{-0.06}^{+0.06}$ & $1_{-0.6}^{+1}$ \\
\hline S82X 004003.87+005853.9 & $287 \mathrm{X}$ & 20.62 & 15.92 & 4.24 & 1.37 & 0.811 & $7.66 \times 10^{44}$ & $-0.47_{-0.53}^{+0.54}$ & $<3$ \\
\hline S82X $004341.18+005253.2$ & $367 X$ & 19.45 & 14.34 & 4.65 & 0.33 & 0.828 & $2.17 \times 10^{44}$ & $-0.09_{-0.17}^{+0.14}$ & $2_{-1.4}^{+1}$ \\
\hline
\end{tabular}

Notes.

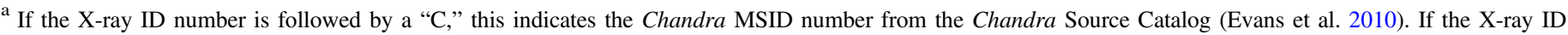
number is appended with an "X," this denotes the XMM-Newton record number introduced in the Stripe 82X survey (LaMassa et al. 2013a, 2016c).

${ }^{\mathrm{b}} k$-corrected (i.e., rest-frame), non-absorption-corrected luminosities.

c $H R=(H-S) /(H+S)$, where $H(S)$ are net counts in the hard (soft) X-ray bands. Hardness ratios were calculated with BEHR.

${ }^{\mathrm{d}}$ Gas column density $\left(N_{\mathrm{H}}\right)$ implied by the $H R$ ranges.
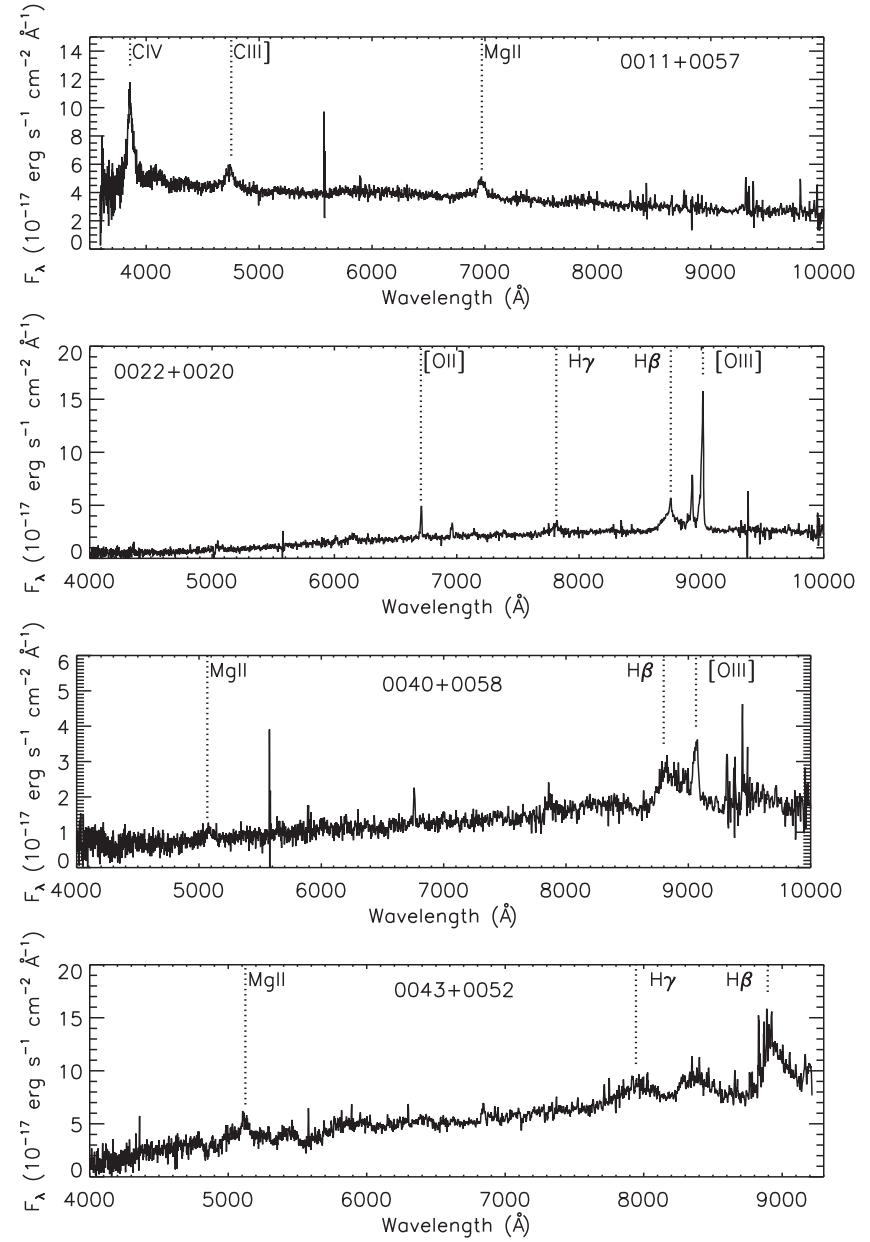

Figure 7. SDSS spectra of extragalactic Stripe $82 X$ sources that meet the selection criteria of our bright NIR Stripe $82 \mathrm{X}$ sample, i.e., $R-K>4, X / O>0$, and $K \leqslant 16$.

AGN power-law continuum with $R=3.1$, and (4) Gaussian components to fit emission lines. The spectra were corrected for Galactic reddening, shifted to the rest frame, and the flux was multiplied by $(1+z)$ to preserve the observed integrated line flux. During the fitting procedure, all narrow emission lines were forced to have the same FWHM, and the [O III] $4959 \AA$ intensity was fixed to a third of the [O III] $5007 \AA$ flux.

Since $\mathrm{H} \beta$ is blended with the [O III] doublet and Fe II emission in S82X 0040+0058, we use the Mg II FWHM $\left(1500 \pm 500 \mathrm{~km} \mathrm{~s}^{-1}\right.$; see Figure 11) and the virial relation above to estimate $M_{\mathrm{BH}}$. However, due to the large errors in $\lambda L_{3000}$ and the Mg II FWHM, we were only able to estimate a $3 \sigma$ upper limit on the black hole mass.

We used the $\mathrm{H} \beta$ FWHM to derive a black hole mass for S82X $0022+0020$. With a fitted FWHM of $5100 \pm 300 \mathrm{~km} \mathrm{~s}^{-1}$ (see Figure 12, top), and using

$$
\begin{aligned}
M_{\mathrm{BH}}= & 1.05 \times 10^{8}\left[\frac{\mathrm{FWHM}(\mathrm{H} \beta)}{1000 \mathrm{~km} \mathrm{~s}^{-1}}\right]^{2} \\
& \times\left[\frac{\lambda L_{5100}}{10^{46} \mathrm{erg} \mathrm{s}^{-1}}\right]^{0.65} M_{\odot}
\end{aligned}
$$

from Trakhtenbrot \& Netzer (2012), we find $M_{\mathrm{BH}}=8.75_{-0.18}^{+0.13} M_{\odot}$, with an associated $\lambda_{\text {Edd }}$ of $0.10 \pm 0.06$.

Trakhtenbrot \& Netzer (2012) do not provide formal errors on the $M_{\mathrm{BH}}-\mathrm{Mg}$ II or $M_{\mathrm{BH}}-\mathrm{H} \beta$ virial relations. Typical standard deviations in the samples used for their calibrations range from $\sim 0.13$ to 0.15 dex. When estimating black hole masses using the Mg II and $\mathrm{H} \beta$ emission FWHMs, we only propagated formal errors on the fit parameters and bolometric corrections, and note that there is likely an additional uncertainty of up to $\sim 0.15$ dex as well as a $\sim 0.5$ dex uncertainty associated with single-epoch black hole mass measurements (Vestergaard \& Peterson 2006).

\subsection{Absorption Line System and Asymmetric Line Profiles: Indications of Outflows?}

Half of the sources from the bright NIR $R-K$ versus $X / O$ selected sample have spectroscopic signatures of narrow-line region kinematics, with either absorption line troughs or broadened [O III] emission: S82X 0043+0052 (Figure 7, bottom), S82X 0022+0020 (Figure 12), S82X 0040+0058 (Figure 11), and S82X 0242+0005 (Figure 6). None of the spectra from the faint NIR WISE-selected optical dropout sample show any sign of outflowing gas, though this sample is only $\sim 12 \%$ complete.

S82X $0043+0052$ was identified as a $\mathrm{Mg}$ II quasar narrow absorption line (FWHM $\leqslant 500 \mathrm{~km} \mathrm{~s}^{-1}$ ) system in Lundgren et al. (2009). Such absorption line systems can be associated with quasar outflows or from gas within the quasar environment (Weymann et al. 1979; Vanden Berk et al. 2008). For the remaining three sources, we found asymmetries in the [O III] $5007 \AA$ line from our own fits to the spectra, as detailed below and summarized in Table 8. In all cases, the widths of the [O III] doublet lines were tied together, the flux of the [O III] 

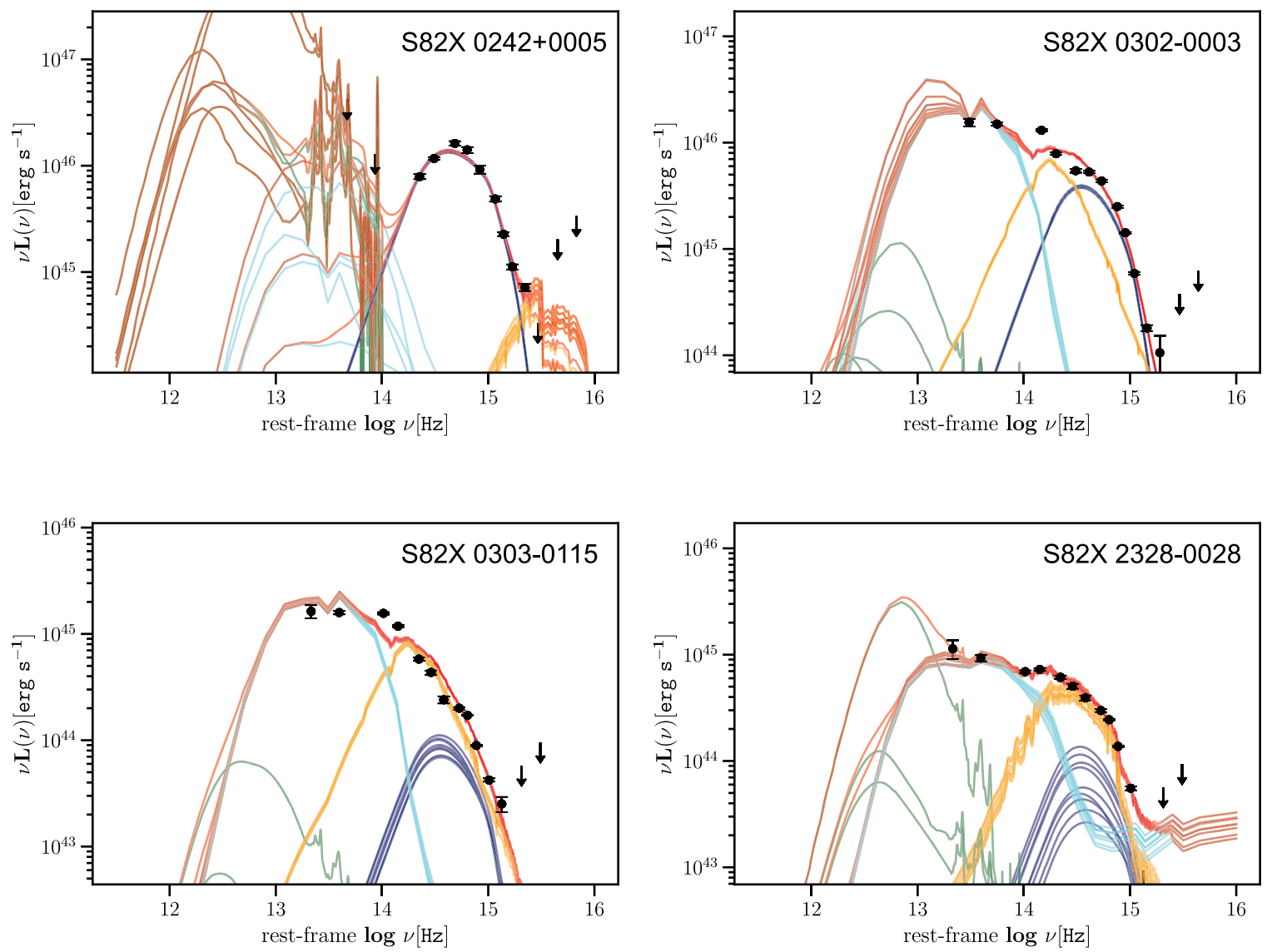

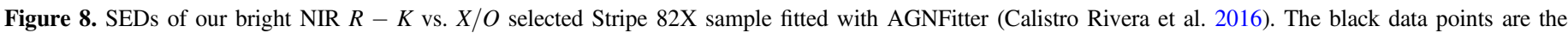

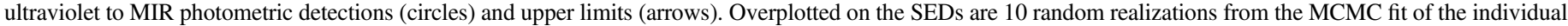

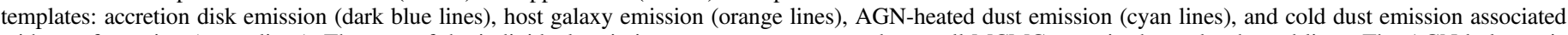

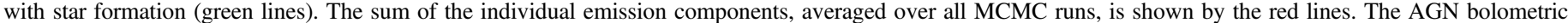
luminosity is based on the integrated luminosity from the de-reddened accretion disk template (i.e., dark blue lines) integrated from 0.03 to $1 \mu$ m.

$4959 \AA$ line was fixed to one-third of the [O III] $5007 \AA$ line, and the central wavelength of the [O III] $4959 \AA$ line was fixed to 0.99 of the [O III] $5007 \AA$ line.

In S82X $0040+0058, \mathrm{H} \beta, \mathrm{Fe}$ II emission, and the [O III] doublet are blended (Figure 11). To fit the spectrum, we include an optical Fe II emission template (Boroson \& Green 1992) as well as broad components to the [O III] doublet. The broad $\mathrm{H} \beta$ emission $\left(\mathrm{FWHM}=6100 \pm 600 \mathrm{~km} \mathrm{~s}^{-1}\right)$ is redshifted with respect to the narrow component $\left(\Delta v=1400 \mathrm{~km} \mathrm{~s}^{-1}\right)$, while the broad [O III] component (FWHM $=2400 \pm 200 \mathrm{~km} \mathrm{~s}^{-1}$ ) is blueshifted compared to the fitted wavelength of the narrow component $\left(\Delta v=-500 \mathrm{~km} \mathrm{~s}^{-1}\right)$. Shifted broad $\mathrm{H} \beta$ emission is sometimes observed in double-peaked emitters with asymmetric line profiles (Eracleous \& Halpern 1994, 2003; Barrows et al. 2011). This feature is typically explained by asymmetries in a Keplerian accretion disk. We note that similar signatures, i.e., high-velocity shifts in the broad $\mathrm{H} \beta$ line, can also be produced by supermassive black hole binaries (Eracleous et al. 2012; Runnoe et al. 2015) and rapidly recoiling black holes (Bonning et al. 2007; Komossa 2012).

An apparent blue wing to the [O III] doublet is present in S82X 0022+0020, which we are able to fit with broad Gaussian components in addition to narrow Gaussians to fit the narrowline doublet (Figure 12). The broad component of the [O III] line has an FWHM of $1200 \pm 200 \mathrm{~km} \mathrm{~s}^{-1}$ and is blueshifted with respect to the narrow component $\left(\mathrm{FWHM}=560 \pm 20 \mathrm{~km} \mathrm{~s}^{-1}\right.$ ) by $\Delta v=-700 \mathrm{~km} \mathrm{~s}^{-1}$.
The Palomar spectrum of S82X $0242+0005$ shows a blue wing to the [O III] doublet. As shown in Figure 6, additional Gaussian components, with FWHM $=2300 \pm 200 \mathrm{~km} \mathrm{~s}^{-1}$, accommodates this additional emission. It is blueshifted by $\Delta v=-400 \mathrm{~km} \mathrm{~s}^{-1}$ compared with the narrow component of the line.

We note that these [O III] FHWM values and velocities are on the order of those observed in $X M M$-COSMOS reddened quasars (Brusa et al. 2015a), but less extreme than the SDSSselected luminous reddened $\left(r_{\mathrm{AB}}-W 4_{\mathrm{Vega}}>14\right)$ quasars (Ross et al. 2015) presented in Zakamska et al. (2016).

\section{Discussion}

\subsection{AGN Properties Derived from Spectral Analysis and SED Fitting}

Two of the sources from the NIR faint optical dropout sample, S82X 0141-0017 and S82X 0227+0042, have estimated bolometric luminosities on the order of, or lower than, the observed full-band X-ray luminosity. This apparent inconsistency points to limitations in the SED decomposition due to the relatively few photometric detections for these sources. We therefore refrain from estimating their black hole masses and Eddington parameters, and note that their $E(B-V)_{\mathrm{AGN}}$ and $E(B-V)_{\text {Galaxy }}$ values may also be unreliable. We therefore discard these objects when considering the AGN properties derived from SED fitting below. 

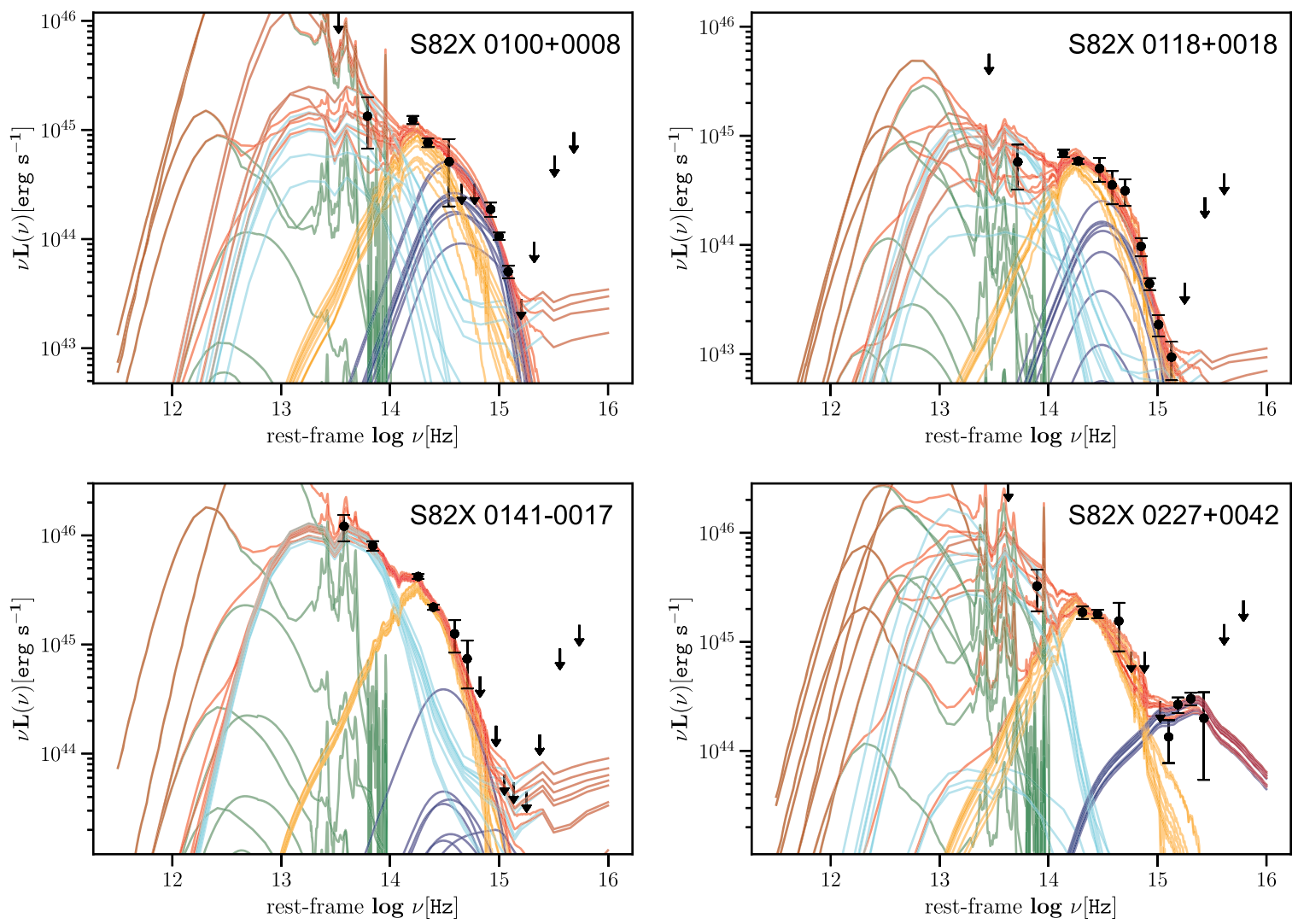

Figure 9. SEDs of our faint NIR WISE-selected optical dropout Stripe 82X sample fitted with AGNFitter (Calistro Rivera et al. 2016). Colors are the same as in Figure 8.

Based on the fitted $E(B-V)$ values from the SED decomposition, nine out of the remaining 10 sources are "reddened" Type 1 AGNs, with $E(B-V) \geqslant 0.45$. We note that the blue source is S82X $0011+0057$, which we showed to be radio loud (Section 4.4). The SDSS spectrum for this source (Figure 7) also shows a blue power-law slope, consistent with an unobscured quasar. Thus, we conclude that the red $R-K$ color for S82X $0011+0057$ is due to synchrotron emission boosting the $K$-band flux and the low $E(B-V)$ value is to be expected. We point out, however, that S82X 0302-0003, which is radio intermediate, appears to be truly reddened based on the $E(B-V)$ values derived from SED decomposition. For the reddened sources, the extinction is along the line of sight to the AGN.

The black hole masses and Eddington ratios span a range of values, with $M_{\mathrm{BH}}-\lambda_{\mathrm{Edd}}$ relationships similar to unobscured quasars from SDSS (Trakhtenbrot \& Netzer 2012). However, we reiterate that the bolometric and monochromatic luminosities from which we derive these values are approximate. Furthermore, obscuration in the broad-line region can skew the emission-line FWHM, which results in systematically lower $M_{\mathrm{BH}}$ estimates compared with unobscured quasars, such that a comparison between both populations is not straightforward.

\subsection{Bright NIR Reddened AGNs are Less Numerous and More Luminous Than Blue Type 1 AGNs}

Glikman et al. $(2007,2012,2013)$ analyzed the properties of radio-selected reddened quasars, finding that their observed surface space density was $\sim 17 \%-21 \%$ lower than a matched sample of radio-selected blue quasars. They also reported that after correcting the $K$-band magnitude for reddening, these red quasars were more luminous than their unobscured counterparts. Similarly, Banerji et al. (2015) and Assef et al. (2015) found that reddened quasars selected on the basis of red NIR colors $(J-K>2.5, K<16.5$, Vega) and red WISE colors, respectively, have higher bolometric luminosities than blue Type 1 AGNs culled from SDSS.

Since our bright NIR $R-K$ sample is $\sim 89 \%$ complete (only one source that fits our selection criteria lacks a spectroscopic redshift; Figure 1), we compare the properties of these reddened AGNs with a matched sample of X-ray-selected blue $(R-K<3)$ Type 1 AGNs, also drawn from Stripe 82X. This comparison sample obeys the same infrared and optical magnitude cuts as the $R-K$ versus $X / O$ sample: $X / O>0, K<16$ (Vega). We discard all sources spectroscopically identified as stars or galaxies (i.e., they lack broad lines in their optical spectra). For sources that lack a redshift, we discarded objects that lie along the $R-K$ versus $R-W 1$ stellar locus (Figure 6 of LaMassa et al. 2016a), so that the comparison sample is made up of likely extragalactic sources. There are 62 such blue sources for comparison, 56 of which have spectra and are confirmed Type 1 AGNs.

First, we calculated the observed surface density for both the reddened and comparison blue AGN samples in X-ray flux bins with a width of 0.3 dex. To account for spectroscopic incompleteness, we multiplied the observed space density (i.e., $N / 31.3 \mathrm{deg}^{2}$ ) within each bin by the fraction of sources spectroscopically identified in that bin. Figure 13 shows the observed space density for the reddened and blue AGNs, where the errors are Poissonian $(\sqrt{N})$ (if there are 10 or more sources in the bin) or are derived from Gehrels (1986). From this exercise, we find that blue Type 1 AGNs have a higher space density than the reddened AGNs, and that they have higher 

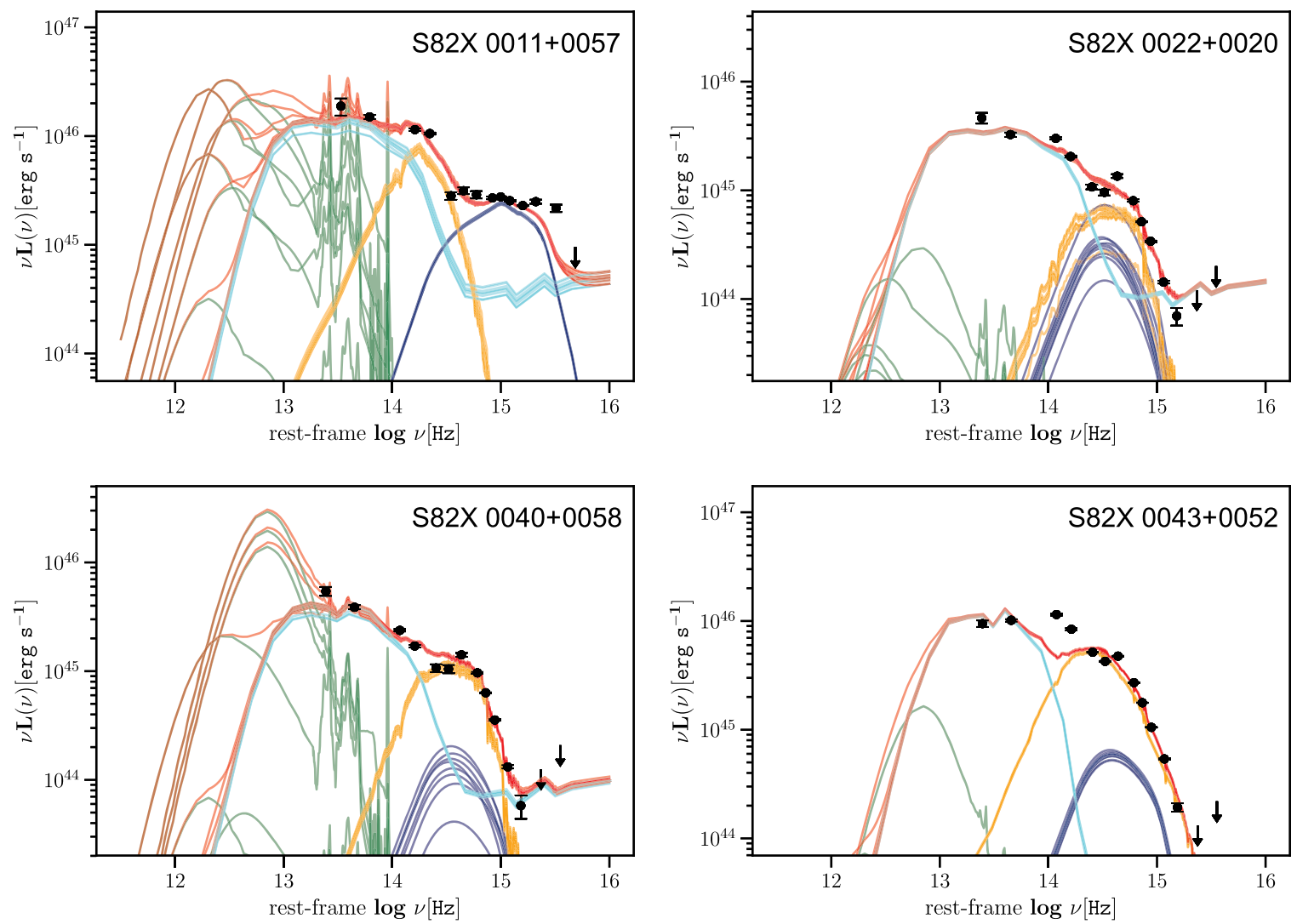

Figure 10. SEDs of our Stripe 82X R-K selected AGN with existing SDSS spectra fitted with AGNFitter (Calistro Rivera et al. 2016). Colors are the same as in Figure 8.

$\mathrm{X}$-ray fluxes than the reddened population. There are no blue Type 1 AGNs at X-ray fluxes below $10^{-13} \mathrm{erg} \mathrm{s}^{-1} \mathrm{~cm}^{-2}$, while the reddened AGNs have a roughly constant space density $\left(\sim 0.06 \mathrm{deg}^{-2}\right)$ as a function of observed X-ray flux.

The blue AGNs extend to brighter X-ray fluxes because they are predominantly nearby compared with the reddened population. As shown in Figure 14 (left), most of the blue AGNs $(66 \%)$ reside at $z<0.5$ while all of the reddened AGNs are more distant. Furthermore, the X-ray luminosities of the reddened population are drawn from the higher end of those observed in the blue AGN population, as illustrated in Figure 14 (right), where we also show the estimated intrinsic $\mathrm{X}$-ray luminosity for the reddened AGNs for reference. The mean X-ray luminosities of both the reddened $\left(\log \left(L_{\mathrm{X}}\right.\right.$,full $\left./ \mathrm{erg} \mathrm{s}^{-1}\right)=44.7 \pm 0.4$ (observed) and $45.0 \pm 0.4$ (intrinsic)) and blue $\left(\log \left(L_{\mathrm{X}, \text { full }} / \mathrm{erg} \mathrm{s}^{-1}\right)=44.8 \pm 0.5\right)$ AGNs are consistent. However, under half of the blue AGNs have observed X-ray luminosities exceeding $10^{44} \mathrm{erg} \mathrm{s}^{-1}$, while $67 \%$ of the reddened AGNs are at these high $\mathrm{X}$-ray luminosities.

Focusing on reddened AGNs in a flux-limited X-ray sample favors detection of AGNs that are more distant, and more luminous, than their unreddened counterparts. This bias is induced by the red $R-K$ criterion and the $K$-band flux limit: sources that are more reddened are those where the AGN dominates over the host galaxy, which are preferentially highluminosity AGNs since lower luminosity AGNs would fall below the $K$-band flux limit. Furthermore, wide-area coverage is required to identify this luminous population at a relatively bright NIR flux limit. We find no X-ray $\mathrm{AGNs}$ (i.e., $L_{\mathrm{X}, \text { full }}>10^{42} \mathrm{erg} \mathrm{s}^{-1}$ ) from the smaller $2.2 \mathrm{deg}^{2}$ Chandra COSMOS Legacy survey (Civano et al. 2016; Marchesi et al.
2016) with the same colors $(R-K>4$, Vega; $X / O>0)$ at the same magnitude limit (i.e., $K<16$, Vega). ${ }^{32}$

\subsection{Stripe $82 X$ Reddened AGNs Compared with Those Previously Known}

We compare our Stripe $82 \mathrm{X}$ reddened quasars with samples from the literature selected based on radio emission and red optical-infrared colors (Glikman et al. 2007, 2012, 2013 $R-K>4, J-K>1.7$, Vega;), NIR colors $(J-K>$ 2.5, $K<16.5$, Vega; Banerji et al. 2012, 2013, 2015), MIR colors (W1W2 dropouts; Eisenhardt et al. 2012; Assef et al. $2015)$, and reddened $(R-K>4-4.5$, Vega) X-ray-selected AGNs presented in Bongiorno et al. (2014).

Although these previous samples of reddened AGNs have been selected via independent methods, there are several traits that many of these sources have in common: the extinction ranges from moderate (i.e., $0.1<E(B-V)<1.55$; Glikman et al. 2007, 2012, 2013; Banerji et al. 2012, 2013, 2015) to extreme (i.e., $2.5<E(B-V)<21.5$; Assef et al. 2015), the black holes are massive $\left(M_{\mathrm{BH}}>10^{9} M_{\odot}\right.$; Bongiorno et al. 2014; Banerji et al. 2015; Wu et al. 2017), the AGNs are generally distributed beyond $z>1$ (Bongiorno et al. 2014) and $z>2$ (Banerji et al. 2012, 2013, 2015; Assef et al. 2015), and they tend to be more luminous than blue Type 1 quasars at comparable redshifts (Glikman et al. 2007, 2012, 2013; Assef

\footnotetext{
32 To match the magnitude system used in this study, we converted the COSMOS $r$-band magnitude from Subaru SuprimeCam reported in the PSFhomogenized photometric catalog of Laigle et al. (2016) to the SDSS $r$-band filter using the formula in Capak et al. (2007). We then transformed to the Bessel $R$ bandpass and converted to the Vega magnitude system using Equations (1) and (2) above.
} 
Table 7

AGN Parameters Derived from SED and Spectral Fitting

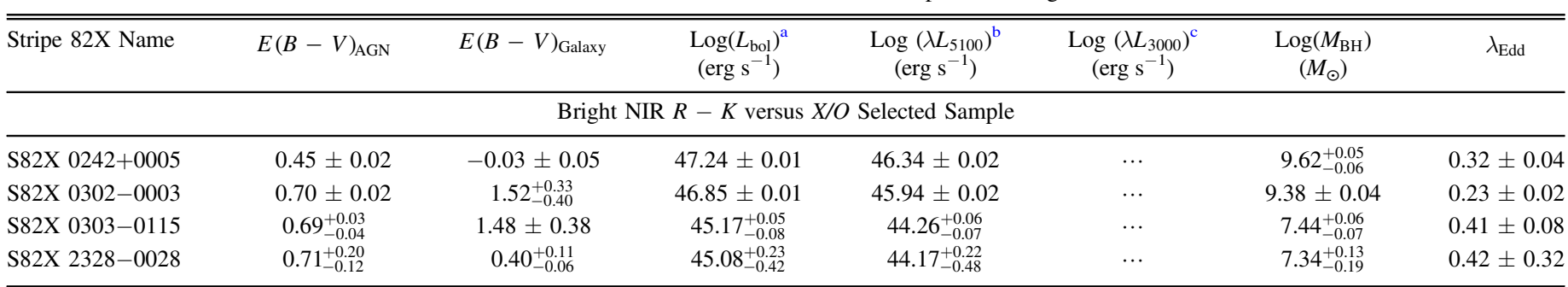

Faint NIR WISE-selected Optical Dropout Sample

\begin{tabular}{|c|c|c|c|c|c|c|c|}
\hline S82X 0100+0008 & $0.54 \pm 0.09$ & $1.50_{-0.44}^{+0.38}$ & $45.52 \pm 0.22$ & $44.61_{-0.32}^{+0.18}$ & $\cdots$ & $8.43_{-0.62}^{+0.25}$ & $0.10 \pm 0.09$ \\
\hline S82X 0118+0018 & $1.18_{-0.41}^{+0.54}$ & $1.20_{-0.49}^{+0.55}$ & $45.41_{-0.99}^{+0.24}$ & $44.50_{-0.76}^{+0.26}$ & $\cdots$ & $7.99_{-0.31}^{+0.18}$ & $\cdots$ \\
\hline S82X 0141-0017 & $1.21_{-0.65}^{+0.51}$ & $1.38_{-0.31}^{+0.41}$ & $44.59_{-0.44}^{+0.67}$ & $\cdots$ & $\cdots$ & $\cdots$ & $\cdots$ \\
\hline $\mathrm{S} 82 \mathrm{X} 0227+0042^{\mathrm{d}}$ & $-0.03 \pm 0.05$ & $0.97_{-0.52}^{+0.70}$ & $44.70_{-0.09}^{+0.06}$ & $\ldots$ & $\ldots$ & $\ldots$ & $\cdots$ \\
\hline
\end{tabular}

Bright NIR $R-K$ versus $X / O$ Selected Sample from SDSS

\begin{tabular}{|c|c|c|c|c|c|c|c|}
\hline S82X $0011+0057^{\mathrm{e}}$ & $0.10 \pm 0.02$ & $1.43_{-0.37}^{+0.39}$ & $46.02 \pm 0.01$ & .. & $45.31 \pm 0.02$ & $8.85_{-0.18}^{+0.13}$ & $0.11 \pm 0.04$ \\
\hline S82X $0022+0020$ & $0.85_{-0.07}^{+0.47}$ & $0.03_{-0.08}^{+0.04}$ & $45.85_{-0.34}^{+0.15}$ & $44.94_{-0.29}^{+0.17}$ & $\cdots$ & $8.75_{-0.18}^{+0.13}$ & $0.10 \pm 0.06$ \\
\hline$S 82 X 0040+0058^{f}$ & $0.63_{-0.13}^{+0.09}$ & $-0.04 \pm 0.04$ & $45.12_{-0.43}^{+0.37}$ & $\ldots$ & $44.41_{-1.72}^{+0.30}$ & $<7.3$ & 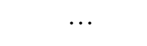 \\
\hline $\mathrm{S} 82 \mathrm{X} 0043+0052^{\mathrm{e}}$ & $0.55 \pm 0.02$ & $1.42_{-0.32}^{+0.39}$ & $45.93_{-0.03}^{+0.04}$ & $\cdots$ & $45.21 \pm 0.04$ & $8.84_{-0.13}^{+0.10}$ & $0.09 \pm 0.03$ \\
\hline
\end{tabular}

Notes.

${ }^{\mathrm{a}} L_{\mathrm{bol}}$ is the AGN bolometric luminosity found by decomposing the SED in AGNFitter and integrating the de-reddened accretion disk luminosity from 0.03 to $1.0 \mu \mathrm{m}$.

b $\lambda L_{5100}$ is the monochromatic continuum luminosity at $5100 \AA$, calculated from $L_{\mathrm{bol}}$ and assuming a bolometric correction of $8.1 \pm 0.4$ (Runnoe et al. 2012 ), which we use to estimate $M_{\mathrm{BH}}$ for sources where $\mathrm{H} \alpha$ or $\mathrm{H} \beta$ is detected (and in the case of $\mathrm{H} \beta$, not blended with the [O III] doublet).

${ }^{c} \lambda L_{3000}$ is the monochromatic continuum luminosity at $3000 \AA$, calculated from $L_{\mathrm{bol}}$ and assuming a bolometric correction of $5.2 \pm 0.2$ (Runnoe et al. 2012 ), which we use to estimate $M_{\mathrm{BH}}$ along with the Mg II FWHM for sources where $\mathrm{H} \alpha$ and $\mathrm{H} \beta$ are not detected or where $\mathrm{H} \beta$ is blended.

${ }^{\mathrm{d}} L_{\mathrm{bol}}$ is on the order of or lower than the observed X-ray luminosity, indicating potential errors in the AGN and galaxy decomposition in the SED fitting. We therefore refrain from calculating $M_{\mathrm{BH}}$ and $\lambda_{\mathrm{Edd}}$, and caution that the $E(B-V)$ values may be unreliable.

e $M_{\mathrm{BH}}$ is estimated using the Mg II FWHM reported in Shen et al. (2011) and $\lambda L_{3000}$ calculated from our SED decomposition (i.e., $\lambda L_{3000}=L_{\mathrm{bol}} /(5.2 \pm 0.2)$ ).

${ }^{\mathrm{f}}$ Due to uncertainties in $\lambda L_{3000}$ and the Mg II FWHM, we report the $3 \sigma$ upper limit on $M_{\mathrm{BH}}$.

et al. 2015; Banerji et al. 2015). Most of the AGNs have broad $\mathrm{H} \alpha$ emission and are not narrow-line only Type 2 AGNs, with the exception of the $W 1 W 2$ dropouts detected by WISE, which are a mixture of Type 1 and Type 2 AGNs (Eisenhardt et al. 2012; Assef et al. 2015).

Glikman et al. (2012) presented a sample of 120 reddened quasar candidates selected from the FIRST radio and 2MASS NIR surveys that have red colors. An analogous sample of radioselected quasar candidates was presented in Glikman et al. (2013), with similar color selection, but pushed down to lower NIR flux limits, using sources detected in the deeper UKIDSS survey. We find that our Stripe $82 \mathrm{X}$ sources span redshift ranges $(0.6<z<2.5)$ similar to those in Glikman et al. (2007, 2012, 2013), $0.13<z<3.1$. We also obtain similar AGN reddening values, where ours have a range of $0.45<E(B-V)<1.18$ (after excluding the radio-loud AGN and the two optical dropout AGNs with inconsistent bolometric and $\mathrm{X}$-ray luminosities) compared with $0.1<E(B-V)<1.55$; our $E(B-V)$ values are derived from SED fitting while those from Glikman et al. $(2007,2012,2013)$ are measured from fitting a reddened quasar template to the optical and/or NIR spectra. Only two of our Stripe $82 \mathrm{X}$ sources are detected in the radio by FIRST, indicating that the orthogonal axis of X-ray selection aids in recovering reddened AGNs not detected by radio surveys.

Similar to Banerji et al. (2015), we estimated $M_{\mathrm{BH}}$ using the FWHM of the broad $\mathrm{H} \alpha$ emission line, where the continuum and bolometric luminosities were calculated via SED fitting. Both samples are subjected to similar broad-line region obscuration biases that could potentially affect emission-line FWHMs. Compared with the $38 z>2$ red quasars presented in Banerji et al. $(2012,2013,2015)$, where $\log \left(M_{\mathrm{BH}} / M_{\odot}\right)=9.7 \pm 0.46$ and $\log \left(L_{\mathrm{bol}} / \mathrm{erg} \mathrm{s}^{-1}\right)=47.1 \pm 0.4$ (Banerji et al. 2015), our X-ray-selected reddened AGNs have lower black hole masses $\left(\log \left(M_{\mathrm{BH}} / M_{\odot}\right)=9.0 \pm 0.8\right)$ and bolometric luminosities $\left(\log \left(L_{\mathrm{bol}} / \mathrm{erg} \mathrm{s}^{-1}\right)=46.5 \pm 0.8\right)$, though there is a wide spread in these values for the Stripe 82X AGNs. Additionally, as the faint NIR optical dropout sample is only $\sim 12 \%$ complete and we are unable to derive estimates of $M_{\mathrm{BH}}$ for half of the sample we have observed, more observations are needed to test whether the pilot sample observed thus far is representative of the parent sample. The measured AGN reddening in the Banerji et al. (2015) sample $(0.5<E(B-V)<1.5)$ spans a range similar to the values calculated in our Stripe 82X sample.

The most luminous, reddened quasars yet identified were selected based on their MIR colors in WISE: these W1W2 dropouts are weak or undetected in WISE bands $W 1$ and $W 2$, but are bright in bands $W 3$ and $W 4$ (Eisenhardt et al. 2012). Assef et al. (2015) analyzed the SEDs of $52 W 1 W 2$ dropouts at $z>1$ and $W 4<7.2$ (Vega) that have Spitzer IRAC data. The reddening in these objects are much more extreme (i.e., $\langle E(B-V)\rangle=6.8)$ than what we observe in the Stripe $82 \mathrm{X}$ reddened AGNs presented here and seen in other reddened AGN samples. The typical bolometric luminosities of the $W 1 W 2$ dropouts, $10^{47}-10^{48} \mathrm{erg} \mathrm{s}^{-1}$, are also much higher than 

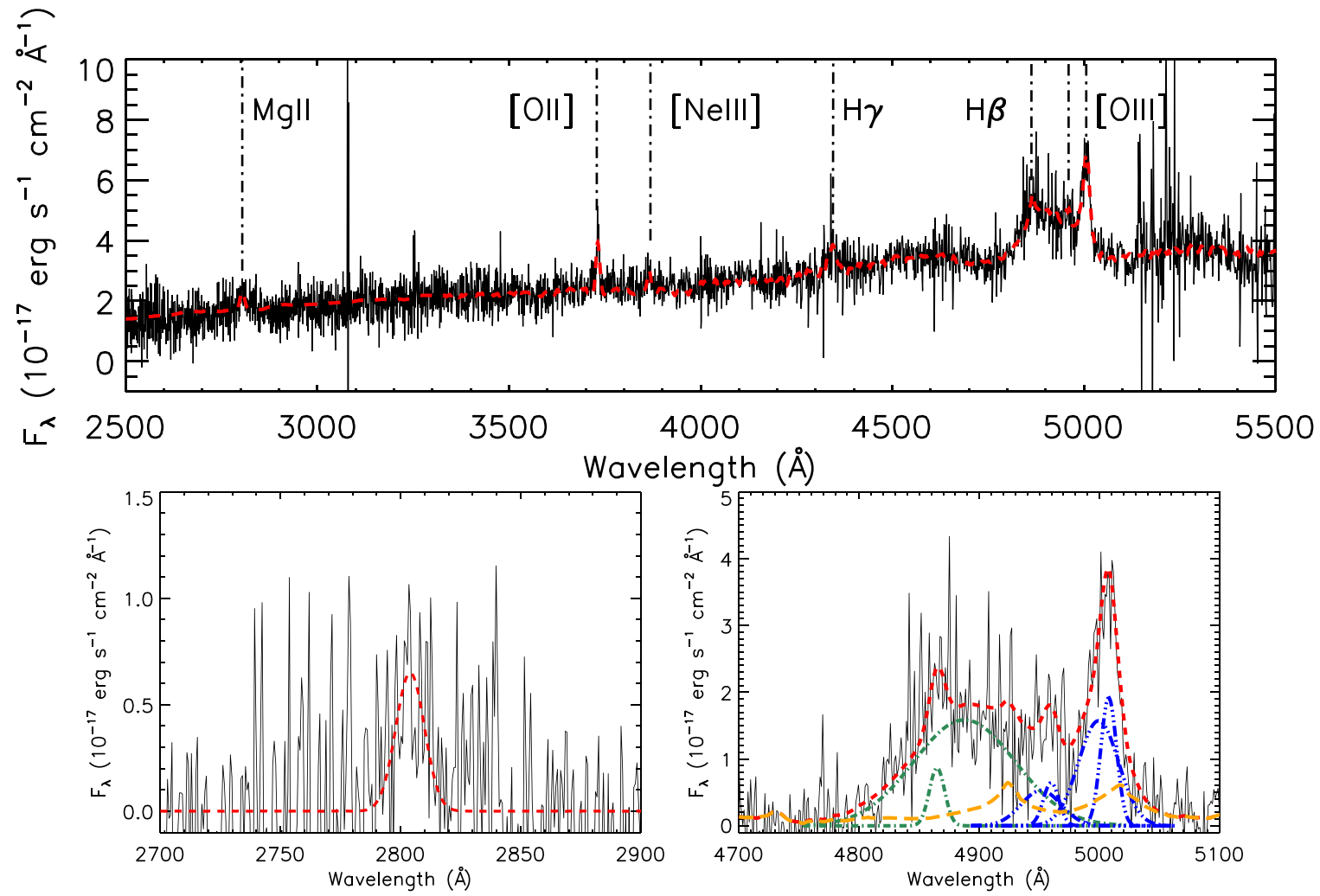

Figure 11. Top: rest-frame SDSS spectrum of S82X 0040+0058 with our best-fit model from IRAF SPECFIT overplotted (red dashed line). Fitted emission lines are marked. Bottom: continuum-subtracted spectra around (left) the Mg II emission line, which we use to derive $M_{\mathrm{BH}}$. The $\mathrm{H} \beta-[\mathrm{O}$ III] complex is shown on the right, where we model the emission lines with an Fe II optical template (long dashed orange lines; Boroson \& Green 1992) and Gaussian profiles for the narrow and broad redshifted $\left(\Delta v=1400 \mathrm{~km} \mathrm{~s}^{1}\right) \mathrm{H} \beta$ lines (green dotted-dashed lines), and narrow and broad blueshifted $\left(\Delta v=-500 \mathrm{~km} \mathrm{~s}^{-1}\right)$ [O III] lines (dotted-dotted-dotteddashed blue line). The red dashed line shows the sum of these emission features.

the Stripe $82 \mathrm{X}$ sample. Three of these sources have been followed up with X-ray observations, with XMM-Newton and NuSTAR, and were found to be X-ray faint, consistent with Compton-thick levels of obscuration (Stern et al. 2014). With our X-ray-optical-infrared selection of reddened quasars, we appear to be selecting an AGN population that is less extreme than the WISE W1W2 dropouts, which, at a space density of $\sim 1 / 30 \mathrm{deg}^{2}$, are rarer than the reddened AGNs selected via other diagnostics.

Finally, we compare our Stripe 82X reddened AGNs with the 21 reddened $(R-K>4.5$, Vega) AGNs from Bongiorno et al. (2014) that were selected from small-to-moderate area X-ray surveys: the original Chandra Deep Field South $\left(0.109 \mathrm{deg}^{2}\right.$; Giacconi et al. 2002), XMM-COSMOS (2 $\mathrm{deg}^{2}$; Hasinger et al. 2007; Cappelluti et al. 2009; Brusa et al. 2010), and the literature (Alexander et al. 2008; Del Moro et al. 2009; Sarria et al. 2010; Melbourne et al. 2011). In this sample, the X-ray emission was mildly absorbed $\left(N_{\mathrm{H}}>10^{21}-10^{22} \mathrm{~cm}^{-2}\right.$, as implied by X-ray spectral analysis or hardness ratios), similar to the implied obscuration of our bright $R-K$ sample. Our faint NIR optical dropout sample, which largely has hardness ratios consistent with no X-ray absorption, spans a similar redshift range as the Bongiorno et al. (2014) sample $(1.2<z<2.6)$. The Bongiorno et al. (2014) sample has a similar average black hole mass $\left(\log \left(M_{\mathrm{BH}} / M_{\odot}\right)=9.3 \pm 0.5\right)$ as the Stripe 82X reddened AGNs. They used the FWHM of the $\mathrm{H} \alpha$ line in conjunction with the intrinsic hard X-ray $(2-10 \mathrm{keV})$ luminosity as a proxy for the AGN continuum luminosity (using the relationship between $\lambda L_{5100}$ and hard X-ray luminosity found in Maiolino et al. 2007) to estimate $M_{\mathrm{BH}}$ :

$$
\begin{aligned}
M_{\mathrm{BH}}= & 10^{7.11}\left[\frac{\mathrm{FWHM}(\mathrm{H} \alpha)}{1000 \mathrm{~km} \mathrm{~s}^{-1}}\right]^{2.06} \\
& \times\left[\frac{\lambda L_{2-10 \mathrm{keV}, \text { intrinsic }}}{10^{44} \mathrm{erg} \mathrm{s}^{-1}}\right]^{0.693} M_{\odot} .
\end{aligned}
$$

Bongiorno et al. (2014) do not provide errors on the parameters in this virial relation, but note that there is a scatter of about 0.1 dex in the normalization.

For reference, in Table 9 we list the black hole masses we obtain using this scaling relation for the four sources that have hard band X-ray detections and $\mathrm{H} \alpha$ coverage. We propagate the errors on the $\mathrm{H} \alpha$ FWHM and $L_{2-10 \mathrm{keV} \text {,intrinsic values and }}$ note that there is likely an additional $\sim 0.1$ dex uncertainty in $M_{\mathrm{BH}}$ that is associated with this virial relation as well as a general $\sim 0.5$ dex uncertainty that is found for single-epoch measurements, as discussed above (Vestergaard \& Peterson 2006). We obtain similar black hole masses compared with what we calculated using $\lambda L_{51000}$ as the continuum luminosity, although the intrinsic hard X-ray luminosities are based on column densities derived from hardness ratios, which are a very crude measure of absorption.

\section{Conclusions}

We presented the results of a ground-based, NIR spectroscopic campaign to follow up reddened AGN candidates in the wide-area $\left(31 \mathrm{deg}^{2}\right)$ Stripe $82 \mathrm{X}$-ray survey (LaMassa et al. 2013a, 2013b, 2016c). Our bright NIR sample selected on the 


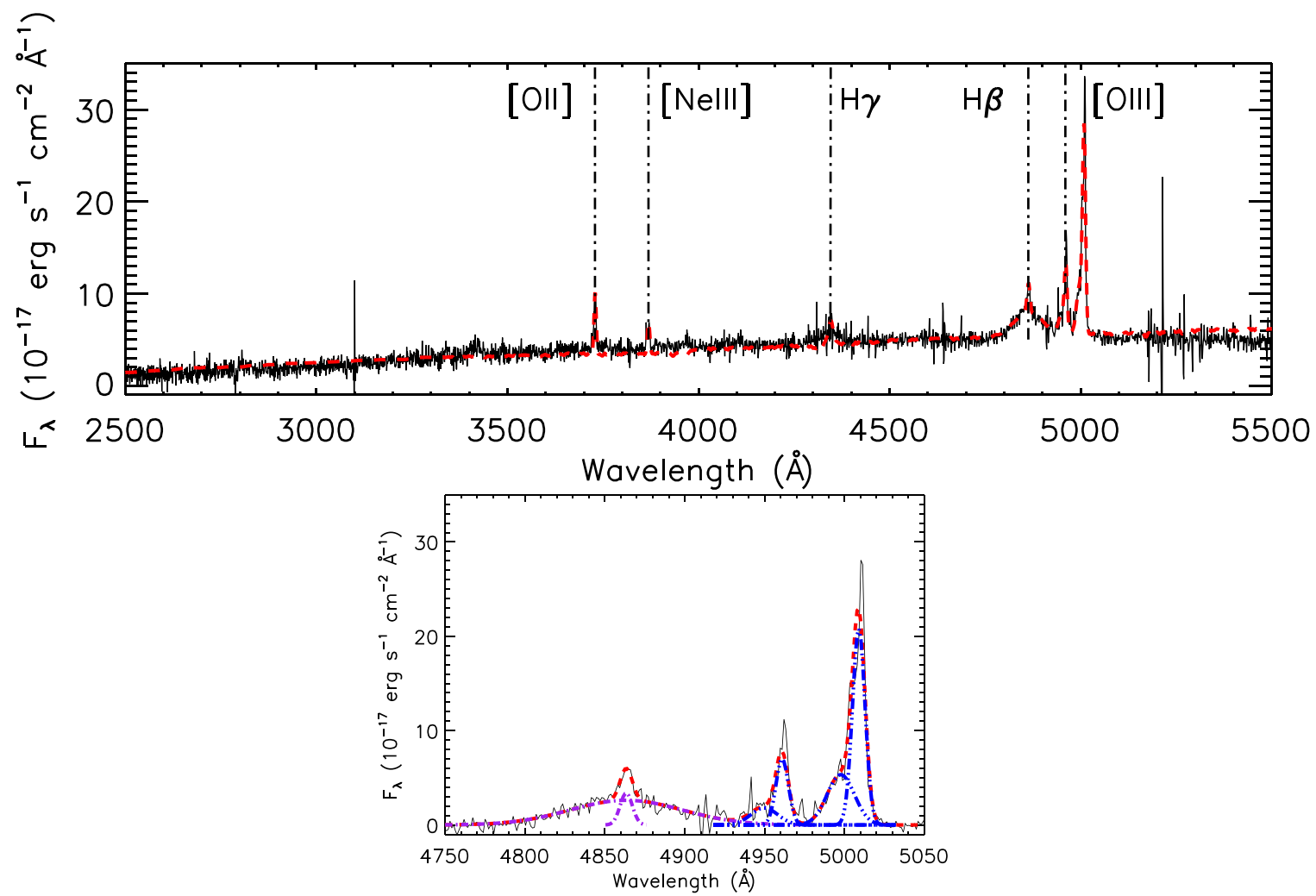

Figure 12. Top: rest-frame SDSS spectrum of S82X 0022+0020 with our best-fit model from IRAF SPECFIT overplotted (red dashed line). Fitted emission lines are marked. Bottom: close-up of the $\mathrm{H} \beta$ and [O III] complex for S82X 0022+0020. Here, the continuum (from the AGN and host galaxy) has been subtracted off. Overplotted are the sum of the fitted emission lines (red dashed line): broad and narrow $\mathrm{H} \beta$ emission lines (dotted-dashed purple line) and [O III] emission (dotteddotted-dotted-dashed blue line), including the narrow and broad (FWHM $=1100 \pm 100 \mathrm{~km} \mathrm{~s}^{-1}$ ) blueshifted $\left(\Delta v=-740 \pm 50 \mathrm{~km} \mathrm{~s} \mathrm{~s}^{-1}\right)[\mathrm{O}$ III] components. This blue wing to the [O III] doublet is likely a signature of an AGN outflow.

Table 8

Asymmetric [O III] Line Profiles

\begin{tabular}{lccl}
\hline \hline Stripe 82X Name & $\begin{array}{c}\text { FWHM } \\
\left(\mathrm{km} \mathrm{s}^{-1}\right)\end{array}$ & $\begin{array}{c}\Delta v^{\mathrm{a}} \\
\left(\mathrm{km} \mathrm{s}^{-1}\right)\end{array}$ & \multicolumn{1}{c}{ Spectrum } \\
\hline S82X 0022+0020 & $1200 \pm 200$ & -700 & SDSS \\
S82X 0040+0058 & $2400 \pm 200$ & -500 & SDSS \\
S82X 0242+0005 & $2300 \pm 200$ & -400 & Palomar TSpec \\
\hline
\end{tabular}

Note.

a $\Delta v$ is measured between the fitted wavelengths of the broad and narrow components of the [O III] $5007 \AA$ line.

basis of red $R-K$ colors $(>4$, Vega) and $X / O>0$ (cf., Brusa et al. 2010) consists of nine sources, four of which had existing spectroscopy in SDSS and five of which we targeted with Palomar TripleSpec (Figure 1); four of the targeted sources were identified via spectroscopic redshifts (Figure 3). This sample is $89 \%$ complete to a magnitude limit of $K=16$ (Vega).

We also presented a pilot program to follow up sources that are not detected in the single-epoch SDSS imaging, yet have WISE colors consistent with quasars (Figure 2; Wright et al. 2010). The spectra of these four sources were obtained with Keck NIRSPEC and Gemini GNIRS since 8-10 m class telescopes are required to spectroscopically identify sources at these faint NIR magnitudes (i.e., $K>17$, Vega; Figure 4).

All sources have at least one permitted emission line with FWHM exceeding $1300 \mathrm{~km} \mathrm{~s}^{-1}$ in their optical or infrared spectra, and can thus be classified as Type 1 AGNs (Hao et al. 2005; Glikman et al. 2007). The bright NIR sample spans a

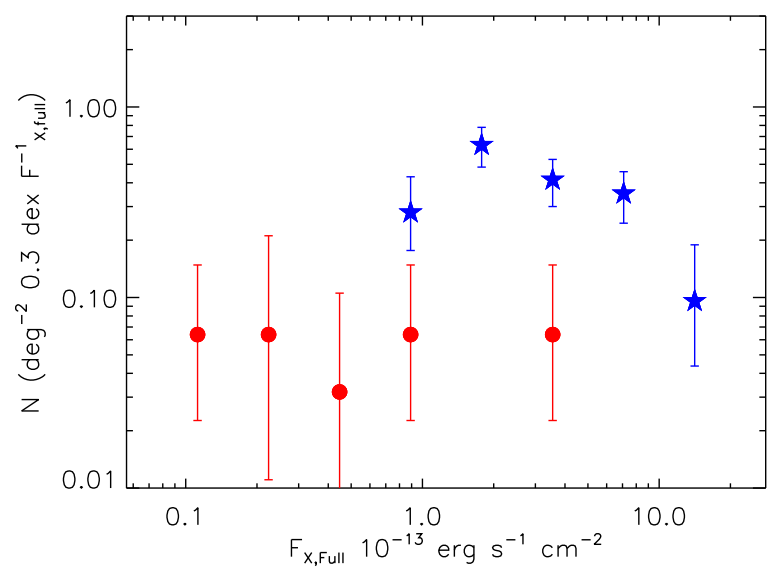

Figure 13. Observed surface space density in bins of 0.3 dex of full-band $X$-ray flux $\left(F_{\mathrm{X} \text {.full }}\right)$ of reddened AGNs from the nearly complete bright NIR $R-K$ vs. $X / O$ selected sample (red circles) compared with a matched sample of X-rayselected blue $(R-K<3)$ Type 1 AGNs (blue stars). Although the space density of the reddened AGNs is relatively constant with X-ray flux, no blue Type 1 AGNs are found at fluxes under $10^{-13} \mathrm{erg} \mathrm{s}^{-1} \mathrm{~cm}^{-2}$.

range of redshifts, $0.59<z<2.5$, while faint optical dropout AGNs all lie beyond a redshift of 1 .

We used AGNFitter (Calistro Rivera et al. 2016) to fit the SEDs and decompose AGN and galaxy emission (Figures 8-10), obtaining estimates of the reddening and AGN bolometric luminosity for each source. Two sources from the optical dropout sample, S82X 0141-0017 and S82X 0027+0042, have estimated $L_{\text {bol }}$ values on the order of or less than the observed X-ray 

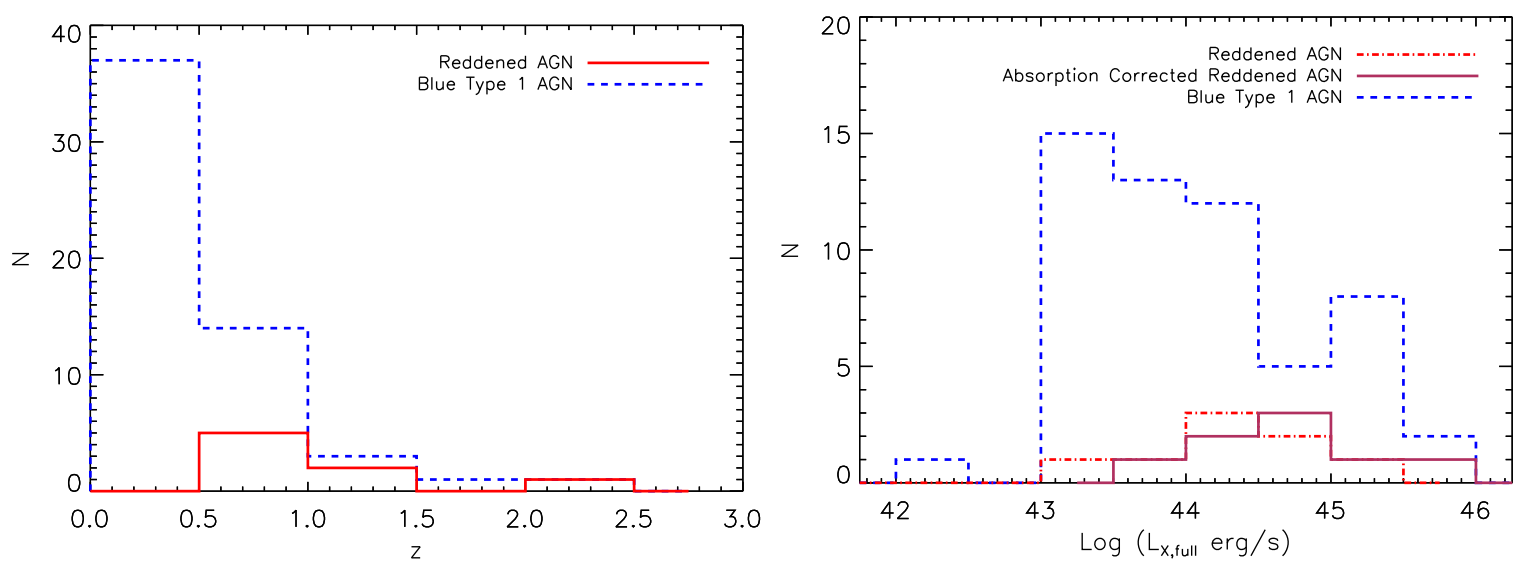

Figure 14. Left: redshift distribution of our bright NIR $R-K$ vs. $X / O$ selected reddened AGNs compared with blue $(R-K<3$, Vega) Type 1 X-ray-selected AGNs from the Stripe $82 \mathrm{X}$ survey at similar magnitude limits (i.e., $K<16$, Vega). The blue AGNs are predominantly at lower redshift $(z<0.5)$ compared with the reddened AGNs. Right: luminosity distribution for the reddened and blue AGN samples, where the estimated intrinsic luminosities, as implied by the hardness ratios, for the reddened AGNs are shown for reference. Though the average luminosities are similar between the reddened and blue populations, a higher fraction of reddened AGNs than blue AGNs have X-ray luminosities exceeding $10^{44} \mathrm{erg} \mathrm{s}^{-1}$.

Table 9

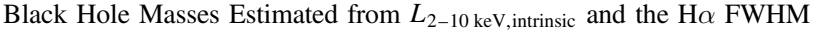

\begin{tabular}{lcc}
\hline \hline Stripe 82X Name & $\begin{array}{c}\log \left(L_{2-10} \mathrm{keV}_{\text {,intrinsic }}\right) \\
\left(\mathrm{erg} \mathrm{s}^{-1} \mathrm{~cm}^{-2}\right)\end{array}$ & $\begin{array}{c}M_{\mathrm{BH}} \\
\left(M_{\odot}\right)\end{array}$ \\
\hline S82X 0242+0005 & $45.05_{-0.31}^{+0.27}$ & $9.26_{-0.29}^{+0.17}$ \\
S82X 0302-0003 & $44.84_{-0.08}^{+0.14}$ & $9.07_{-0.09}^{+0.08}$ \\
S82X 0303-0115 & $43.77_{-0.11}^{+0.11}$ & $7.29_{-0.09}^{+0.07}$ \\
S82X 0118+0018 & $44.12_{-0.00}^{+0.14}$ & $7.98_{-0.26}^{+0.16}$ \\
\hline
\end{tabular}

Note.

${ }^{\text {a }}$ Since we only have an upper limit on the estimated $N_{\mathrm{H}}$, the lower limit on the intrinsic luminosity is the observed luminosity.

luminosity, suggesting limitations in the SED decomposition for these sources. All but one of the remaining AGNs are reddened, with $0.45<E(B-V)_{\mathrm{AGN}}<1.18$. The blue source is radio loud, such that its red $R-K$ color is likely due to jet-dominated synchrotron emission.

Half of the sources in the bright NIR sample have features in their optical spectra indicative of outflows (Figure 6, left; Figure 7, bottom; Figure 11, bottom right; and Figure 12 bottom). Since many quasars host outflows (e.g., Ganguly \& Brotherton 2008), follow-up high-resolution imaging of the host galaxies would be necessary to search for morphological signatures of mergers to test whether these features signify feedback predicted by the major merger AGN evolution paradigm (Sanders et al. 1988; Hopkins et al. 2008).

Because the bright NIR sample is nearly complete, we compared the characteristics of these AGNs with blue $(R-K<3$, Vega) Type 1 AGNs selected from the Stripe $82 \mathrm{X}$ survey. Although the blue Type 1 AGNs have systematically higher X-ray fluxes (Figure 13), they are predominantly at low redshift $(z<0.5)$, with a greater percentage at lower X-ray luminosities compared with the reddened AGNs (Figure 14). Hence, focusing on reddened populations in shallow X-ray surveys, like Stripe $82 \mathrm{X}$, for follow-up will likely unveil more distant and more luminous AGNs than blue AGNs at similar X-ray, optical, and infrared flux limits.

Compared with reddened AGNs selected on the basis of their radio, optical, NIR, and/or MIR emission, the Stripe $82 \mathrm{X}$ red quasars have similar reddening (though less extreme than
$W 1 W 2$ dropouts, which are extreme sources that have the highest levels of extinction compared to all samples of reddened quasars; Assef et al. 2015) and a range of estimated black hole masses and Eddington parameters.

Our pilot sample of WISE-selected optical dropouts is only $\sim 12 \%$ complete, precluding us from drawing any firm conclusions about this population as a whole. Our program does demonstrate proof of concept for using this selection technique to recover reddened quasars at $z>1$ that are missed by optical surveys like SDSS.

We highlight that Stripe $82 \mathrm{X}$ complements other X-ray surveys by discovering reddened AGNs at relatively bright NIR magnitudes (i.e., $K<16$, Vega) that are missed entirely by smaller-area X-ray surveys, like the $2.2 \mathrm{deg}^{2}$ Chandra COSMOS Legacy (Civano et al. 2016; Marchesi et al. 2016). As these sources are rare, potentially due to the reddened stage being a short-lived AGN evolutionary phase, wide-area X-ray surveys like Stripe 82X, XMM-XXL (Pierre et al. 2016), XBoötes (Kenter et al. 2005; Murray et al. 2005), and the upcoming eROSITA mission (Merloni et al. 2012), and serendipitous surveys/catalogs like ChaMP (Kim et al. 2007), the Chandra Source Catalog (Evans et al. 2010), and the XMM Serendipitous catalog (Rosen et al. 2016), are necessary to reveal this missing tier of luminous, obscured black hole growth at the brightest fluxes.

We thank the anonymous referee for a careful reading of this manuscript and providing helpful comments. Most of this work was completed while S.M.L. was supported by an appointment to the NASA Postdoctoral Program at the NASA Goddard Space Flight Center, administered by Universities Space Research Association under contract with NASA. S.M.L. thanks A.-N. Chene for support when running the Gemini GNIRS reduction pipeline and G. Calistro Rivera for guidance in using AGNFitter. Palomar and Keck observations were obtained through guaranteed Yale time on these facilities. E.G. acknowledges the generous support of the Cottrell College Award through the Research Corporation for Science Advancement. The work of D.S. was carried out at the Jet Propulsion Laboratory, California Institute of Technology, under a contract with NASA.

Some of the data presented herein were obtained at the W. M. Keck Observatory, which is operated as a scientific 
partnership among the California Institute of Technology, the University of California, and the National Aeronautics and Space Administration. The Observatory was made possible by the generous financial support of the W. M. Keck Foundation. The authors wish to recognize and acknowledge the very significant cultural role and reverence that the summit of Maunakea has always had within the indigenous Hawaiian community. We are most fortunate to have the opportunity to conduct observations from this mountain.

Facility: XMM, CXO, Sloan, Hale (TSPEC), Gemini:Gillet (GNIRS), Keck:II (NIRSPEC).

\section{ORCID iDs}

Stephanie M. LaMassa (1) https://orcid.org/0000-00025907-3330

Eilat Glikman (1) https://orcid.org/0000-0003-0489-3750

Marcella Brusa (1) https://orcid.org/0000-0002-5059-6848

Jane R. Rigby (1) https://orcid.org/0000-0002-7627-6551

Daniel Stern (i) https://orcid.org/0000-0003-2686-9241

C. Megan Urry (ib https://orcid.org/0000-0002-0745-9792

Mara Salvato (i) https://orcid.org/0000-0001-7116-9303

Rachael Alexandroff (1) https://orcid.org/0000-0003-

2830-0913

Viola Allevato (ํ) https://orcid.org/0000-0001-7232-5152

Carolin Cardamone (i) https://orcid.org/0000-0003-4608-6340

Duncan Farrah 1 https://orcid.org/0000-0003-1748-2010

S. Komossa (ib https://orcid.org/0000-0002-9214-4428

Giorgio Lanzuisi (ib https://orcid.org/0000-0001-9094-0984

Stefano Marchesi iㅏ https://orcid.org/0000-0001-5544-0749

Gordon Richards (i) https://orcid.org/0000-0002-1061-1804

Benny Trakhtenbrot (i) https://orcid.org/0000-0002-

3683-7297

\section{References}

Alexander, D. M., Brandt, W. N., Smail, I., et al. 2008, AJ, 135, 1968 Alexandroff, R., Strauss, M. A., Greene, J. E., et al. 2013, MNRAS, 435, 3306 Ananna, T. T., Salvato, M., LaMassa, S. M., et al. 2017, ApJ, submitted Antonucci, R. 1993, ARA\&A, 31, 473

Assef, R. J., Eisenhardt, P. R. M., Stern, D., et al. 2015, ApJ, 804, 27

Banerji, M., Alaghband-Zadeh, S., Hewett, P. C., \& McMahon, R. G. 2015, MNRAS, 447, 3368

Banerji, M., McMahon, R. G., Hewett, P. C., et al. 2012, MNRAS, 427, 2275

Banerji, M., McMahon, R. G., Hewett, P. C., Gonzalez-Solares, E., \& Koposov, S. E. 2013, MNRAS, 429, L55

Barrows, R. S., Lacy, C. H. S., Kennefick, D., Kennefick, J., \& Seigar, M. S. 2011, NewA, 16, 122

Bassani, L., Dadina, M., Maiolino, R., et al. 1999, ApJS, 121, 473

Bessell, M. S. 1990, PASP, 102, 1181

Blanton, M. R., \& Roweis, S. 2007, AJ, 133, 734

Bongiorno, A., Maiolino, R., Brusa, M., et al. 2014, MNRAS, 443, 2077

Bonning, E. W., Shields, G. A., \& Salviander, S. 2007, ApJL, 666, L13

Boroson, T. A., \& Green, R. F. 1992, ApJS, 80, 109

Brandt, W. N., \& Hasinger, G. 2005, ARA\&A, 43, 827

Brusa, M., Bongiorno, A., Cresci, G., et al. 2015a, MNRAS, 446, 2394

Brusa, M., Civano, F., Comastri, A., et al. 2010, ApJ, 716, 348

Brusa, M., Feruglio, C., Cresci, G., et al. 2015b, A\&A, 578, A11

Brusa, M., Perna, M., Cresci, G., et al. 2016, A\&A, 588, A58

Bruzual, G., \& Charlot, S. 2003, MNRAS, 344, 1000

Calistro Rivera, G., Lusso, E., Hennawi, J. F., \& Hogg, D. W. 2016, ApJ, 833, 98

Capak, P., Aussel, H., Ajiki, M., et al. 2007, ApJS, 172, 99

Cappelluti, N., Brusa, M., Hasinger, G., et al. 2009, A\&A, 497, 635

Cardamone, C. N., Moran, E. C., \& Kay, L. E. 2007, AJ, 134, 1263

Cardelli, J. A., Clayton, G. C., \& Mathis, J. S. 1989, ApJ, 345, 245

Chary, R., \& Elbaz, D. 2001, ApJ, 556, 562

Civano, F., Marchesi, S., Comastri, A., et al. 2016, ApJ, 819, 62
Cooke, A., \& Rodgers, B. 2005, in ASP Conf. Ser. 347, Astronomical Data Analysis Software and Systems XIV, ed. P. Shopbell, M. Britton, \& R. Ebert (San Francisco, CA: ASP), 514

Cushing, M. C., Vacca, W. D., \& Rayner, J. T. 2004, PASP, 116, 362

Dale, D. A., \& Helou, G. 2002, ApJ, 576, 159

Davis, M., Faber, S. M., Newman, J., et al. 2003, Proc. SPIE, 4834, 161

Del Moro, A., Watson, M. G., Mateos, S., et al. 2009, A\&A, 493, 445

Dickey, J. M., \& Lockman, F. J. 1990, ARA\&A, 28, 215

Donley, J. L., Koekemoer, A. M., Brusa, M., et al. 2012, ApJ, 748, 142

Eisenhardt, P. R. M., Wu, J., Tsai, C.-W., et al. 2012, ApJ, 755, 173

Elias, J. H., Joyce, R. R., Liang, M., et al. 2006a, Proc. SPIE, 6269, 62694C

Elias, J. H., Rodgers, B., Joyce, R. R., et al. 2006b, Proc. SPIE, 6269, 626914

Eracleous, M., Boroson, T. A., Halpern, J. P., \& Liu, J. 2012, ApJS, 201, 23

Eracleous, M., \& Halpern, J. P. 1994, ApJS, 90, 1

Eracleous, M., \& Halpern, J. P. 2003, ApJ, 599, 886

Evans, I. N., Primini, F. A., Glotfelty, K. J., et al. 2010, ApJS, 189, 37

Faber, S. M., Phillips, A. C., Kibrick, R. I., et al. 2003, Proc. SPIE, 4841, 1657

Farrah, D., Petty, S., Connolly, B., et al. 2017, ApJ, 844, 106

Farrah, D., Urrutia, T., Lacy, M., et al. 2012, ApJ, 745, 178

Fliri, J., \& Trujillo, I. 2016, MNRAS, 456, 1359

Frank, J., King, A., \& Raine, D. J. 2002, in Accretion Power in Astrophysics, ed. J. Frank, A. King, \& D. Raine (Cambridge: Cambridge Univ. Press), 398

Frieman, J. A., Bassett, B., Becker, A., et al. 2008, AJ, 135, 338

Ganguly, R., \& Brotherton, M. S. 2008, ApJ, 672, 102

Gehrels, N. 1986, ApJ, 303, 336

Georgakakis, A., Clements, D. L., Bendo, G., et al. 2009, MNRAS, 394, 533 Giacconi, R., Zirm, A., Wang, J., et al. 2002, ApJS, 139, 369

Giavalisco, M., Ferguson, H. C., Koekemoer, A. M., et al. 2004, ApJL, 600, L93

Glikman, E., Helfand, D. J., White, R. L., et al. 2007, ApJ, 667, 673

Glikman, E., Simmons, B., Mailly, M., et al. 2015, ApJ, 806, 218

Glikman, E., Urrutia, T., Lacy, M., et al. 2012, ApJ, 757, 51

Glikman, E., Urrutia, T., Lacy, M., et al. 2013, ApJ, 778, 127

Green, P. J., Silverman, J. D., Cameron, R. A., et al. 2004, ApJS, 150, 43

Greene, J. E., Peng, C. Y., \& Ludwig, R. R. 2010, ApJ, 709, 937

Hamann, F., Zakamska, N. L., Ross, N., et al. 2017, MNRAS, 464, 3431

Hao, L., Strauss, M. A., Tremonti, C. A., et al. 2005, AJ, 129, 1783

Hasinger, G., Cappelluti, N., Brunner, H., et al. 2007, ApJS, 172, 29

Heckman, T. M., Kauffmann, G., Brinchmann, J., et al. 2004, ApJ, 613, 109

Heckman, T. M., Ptak, A., Hornschemeier, A., \& Kauffmann, G. 2005, ApJ, 634,161

Helfand, D. J., White, R. L., \& Becker, R. H. 2015, ApJ, 801, 26

Herter, T. L., Henderson, C. P., Wilson, J. C., et al. 2008, Proc. SPIE, 7014, $70140 \mathrm{X}$

Hewett, P. C., Warren, S. J., Leggett, S. K., \& Hodgkin, S. T. 2006, MNRAS, 367, 454

Hopkins, P. F., Hernquist, L., Cox, T. J., et al. 2006, ApJS, 163, 1

Hopkins, P. F., Hernquist, L., Cox, T. J., \& Kereš, D. 2008, ApJS, 175, 356

Hopkins, P. F., Hernquist, L., Martini, P., et al. 2005, ApJL, 625, L71

Hopkins, P. F., Kocevski, D. D., \& Bundy, K. 2014, MNRAS, 445, 823

Ivezić, Ž., Menou, K., Knapp, G. R., et al. 2002, AJ, 124, 2364

Jiang, L., Fan, X., Bian, F., et al. 2014, ApJS, 213, 12

Kenter, A., Murray, S. S., Forman, W. R., et al. 2005, ApJS, 161, 9

Kim, M., Kim, D.-W., Wilkes, B. J., et al. 2007, ApJS, 169, 401

Kocevski, D. D., Brightman, M., Nandra, K., et al. 2015, ApJ, 814, 104

Kocevski, D. D., Faber, S. M., Mozena, M., et al. 2012, ApJ, 744, 148

Komossa, S. 2012, AdAst, 2012, 364973

Krawczyk, C. M., Richards, G. T., Mehta, S. S., et al. 2013, ApJS, 206, 4

Kriss, G. 1994, in ASP Conf. Ser. 61, Astronomical Data Analysis Software and Systems III, ed. D. R. Crabtree, R. J. Hanisch, \& J. Barnes (San Fransisco, CA: ASP), 437

Laigle, C., McCracken, H. J., Ilbert, O., et al. 2016, ApJS, 224, 24

LaMassa, S. M., Civano, F., Brusa, M., et al. 2016a, ApJ, 818, 88

LaMassa, S. M., Heckman, T. M., Ptak, A., et al. 2009, ApJ, 705, 568

LaMassa, S. M., Heckman, T. M., Ptak, A., et al. 2010, ApJ, 720, 786

LaMassa, S. M., Heckman, T. M., Ptak, A., et al. 2011, ApJ, 729, 52

LaMassa, S. M., Ricarte, A., Glikman, E., et al. 2016b, ApJ, 820, 70

LaMassa, S. M., Urry, C. M., Cappelluti, N., et al. 2013a, MNRAS, 436 3581

LaMassa, S. M., Urry, C. M., Cappelluti, N., et al. 2016c, ApJ, 817, 172

LaMassa, S. M., Urry, C. M., Glikman, E., et al. 2013b, MNRAS, 432, 1351

LaMassa, S. M., Yaqoob, T., Ptak, A. F., et al. 2014, ApJ, 787, 61

Lansbury, G. B., Alexander, D. M., Del Moro, A., et al. 2014, ApJ, 785, 17

Lansbury, G. B., Gandhi, P., Alexander, D. M., et al. 2015, ApJ, 809, 115

Lawrence, A., Warren, S. J., Almaini, O., et al. 2007, MNRAS, 379, 1599

Lundgren, B. F., Brunner, R. J., York, D. G., et al. 2009, ApJ, 698, 819 
Maiolino, R., Shemmer, O., Imanishi, M., et al. 2007, A\&A, 468, 979

Marchesi, S., Civano, F., Elvis, M., et al. 2016, ApJ, 817, 34

Markwardt, C. B. 2009, in ASP Conf. Ser. 411, Astronomical Data Analysis Software and Systems XVIII, ed. D. A. Bohlender, D. Durand, \& P. Dowler (San Fransisco, CA: ASP), 251

Martin, D. C., Fanson, J., Schiminovich, D., et al. 2005, ApJL, 619, L1

McLean, I. S., Becklin, E. E., Bendiksen, O., et al. 1998, Proc. SPIE, 3354, 566

McMahon, R. G., Banerji, M., Gonzalez, E., et al. 2013, Msngr, 154, 35

Mechtley, M., Jahnke, K., Windhorst, R. A., et al. 2016, ApJ, 830, 156

Melbourne, J., Peng, C. Y., Soifer, B. T., et al. 2011, AJ, 141, 141

Merloni, A., Predehl, P., Becker, W., et al. 2012, arXiv:1209.3114

Miller, B. P., Brandt, W. N., Schneider, D. P., et al. 2011, ApJ, 726, 20

Morrissey, P., Conrow, T., Barlow, T. A., et al. 2007, ApJS, 173, 682

Murray, S. S., Kenter, A., Forman, W. R., et al. 2005, ApJS, 161, 1

Panessa, F., Bassani, L., Cappi, M., et al. 2006, A\&A, 455, 173

Pâris, I., Petitjean, P., Ross, N. P., et al. 2017, A\&A, 597, A79

Park, T., Kashyap, V. L., Siemiginowska, A., et al. 2006, ApJ, 652, 610

Perna, M., Brusa, M., Cresci, G., et al. 2015, A\&A, 574, A82

Perna, M., Lanzuisi, G., Brusa, M., Mignoli, M., \& Cresci, G. 2017, A\&A, 603, A99

Pierre, M., Pacaud, F., Adami, C., et al. 2016, A\&A, 592, A1

Planck Collaboration, Ade, P. A. R., Aghanim, N., et al. 2016, A\&A, 594, A13

Reyes, R., Zakamska, N. L., Strauss, M. A., et al. 2008, AJ, 136, 2373

Ricci, C., Bauer, F. E., Treister, E., et al. 2017, MNRAS, 468, 1273

Richards, G. T., Lacy, M., Storrie-Lombardi, L. J., et al. 2006, ApJS, 166, 470

Rosen, S. R., Webb, N. A., Watson, M. G., et al. 2016, A\&A, 590, A1

Ross, N. P., Hamann, F., Zakamska, N. L., et al. 2015, MNRAS, 453, 3932

Runnoe, J. C., Brotherton, M. S., \& Shang, Z. 2012, MNRAS, 422, 478

Runnoe, J. C., Eracleous, M., Mathes, G., et al. 2015, ApJS, 221, 7

Sanders, D. B., Soifer, B. T., Elias, J. H., Neugebauer, G., \& Matthews, K. 1988, ApJL, 328, L35

Sarria, J. E., Maiolino, R., La Franca, F., et al. 2010, A\&A, 522, L3
Schawinski, K., Simmons, B. D., Urry, C. M., Treister, E., \& Glikman, E. 2012, MNRAS, 425, L61

Serjeant, S. 1996, Natur, 379, 304

Shen, Y., Richards, G. T., Strauss, M. A., et al. 2011, ApJS, 194, 45

Silva, L., Maiolino, R., \& Granato, G. L. 2004, MNRAS, 355, 973

Stern, D., Lansbury, G. B., Assef, R. J., et al. 2014, ApJ, 794, 102

Trakhtenbrot, B., \& Netzer, H. 2012, MNRAS, 427, 3081

Treister, E., Natarajan, P., Sanders, D. B., et al. 2010, Sci, 328, 600

Treister, E., Schawinski, K., Urry, C. M., \& Simmons, B. D. 2012, ApJL, 758, L39

Urrutia, T., Becker, R. H., White, R. L., et al. 2009, ApJ, 698, 1095

Urrutia, T., Lacy, M., \& Becker, R. H. 2008, ApJ, 674, 80

Urry, C. M., \& Padovani, P. 1995, PASP, 107, 803

Vacca, W. D., Cushing, M. C., \& Rayner, J. T. 2003, PASP, 115, 389

Vanden Berk, D., Khare, P., York, D. G., et al. 2008, ApJ, 679, 239

Vestergaard, M., \& Osmer, P. S. 2009, ApJ, 699, 800

Vestergaard, M., \& Peterson, B. M. 2006, ApJ, 641, 689

Villforth, C., Hamann, F., Rosario, D. J., et al. 2014, MNRAS, 439 3342

Villforth, C., Hamilton, T., Pawlik, M. M., et al. 2017, MNRAS, 466, 812

Weedman, D. W. 1977, ARA\&A, 15, 69

Weymann, R. J., Williams, R. E., Peterson, B. M., \& Turnshek, D. A. 1979, ApJ, 234, 33

Wright, E. L., Eisenhardt, P. R. M., Mainzer, A. K., et al. 2010, AJ, 140, 1868

Wu, J., Jun, H. D., Assef, R. J., et al. 2017, arXiv:1703.06888

Wu, X.-B., Wang, R., Kong, M. Z., Liu, F. K., \& Han, J. L. 2004, A\&A, 424, 793

Yaqoob, T., Tatum, M. M., Scholtes, A., Gottlieb, A., \& Turner, T. J. 2015, MNRAS, 454, 973

Yip, C. W., Connolly, A. J., Vanden Berk, D. E., et al. 2004, AJ, 128, 2603

York, D. G., Adelman, J., Anderson, J. E., Jr., et al. 2000, AJ, 120, 1579

Zakamska, N. L., Hamann, F., Pâris, I., et al. 2016, MNRAS, 459, 3144

Zakamska, N. L., Strauss, M. A., Krolik, J. H., et al. 2003, AJ, 126, 2125 